\title{
ON ACTIONS OF REGULAR TYPE ON COMPLEX STIEFEL MANIFOLDS
}

BY

MCKENZIE Y. WANG ${ }^{1}$

\begin{abstract}
The usual unitary representations of the special unitary, symplectic, or special orthogonal groups define a sequence of smooth actions on the complex Stiefel manifolds called the regular linear models. If one of the above groups acts smoothly on the complex Stiefel manifold of orthonormal 2-frames in $\mathbf{C}^{n}$ for odd $n$, and if the identity component of the principal isotropy type is of regular type, then it is shown under mild dimension restrictions that the orbit structure and the cohomology structure of the fixed point varieties (over the mod 2 Steenrod algebra) resemble those of the regular linear models. The resemblance is complete in the cases of the special unitary and symplectic groups. There is an obstruction to complete resemblance in the case of the special orthogonal groups.

An application of the above regularity theorems is given.
\end{abstract}

Introduction. Let $K / H$ be a compact homogeneous space. Then $K$ acts transitively on $K / H$ by left translations. If $G$ is a compact Lie group, one obtains a family of natural actions on $K / H$ by composing a homomorphism $h: G \rightarrow K$ on the left with the natural transitive action of $K$ on $K / H$. Since when $K$ is a linear Lie group, $h$ is nothing but a linear representation of $G$, we shall refer to actions in the above family as the linear models of $K / H$.

In [12, p. 231] and [14, Chapter VII], W. C. Hsiang and W. Y. Hsiang have suggested the study of arbitrary smooth $G$-actions on $K / H$ by comparison with the linear models on $K / H$. This is a formidable problem in view of the fact that the case $K / H=O(n+1) / O(n)$ alone took well over ten years of work before a reasonably complete understanding was achieved (for example, see $[5,10,11,13,14])$.

Consequently, before the general problem can be approached, it is necessary to study special cases of $K / H$ which cover a large variety of topological types. In a series of papers, we begin to analyse the situation on $W_{n, 2}$ for odd $n$, where $W_{n, 2}=X$ denotes the complex Stiefel manifold of orthonormal 2-frames in $\mathbf{C}^{n}$.

The $W_{n, 2}$ 's were chosen for three reasons: their relation to odd spheres (which can be regarded as $W_{n, 1}$ ), the variety of linear models (as numerous as the number of unitary representations of $G$ ), and the wealth of information on their homotopy [18, 19, 20]. Particular mention should be made of the fact that while the $W_{n, 2}$ 's are of the

\footnotetext{
Received by the editors November 26, 1980 and, in revised form, August 4, 1981, 1980 Mathematics Subject Classification. Primary 57S15.

Key words and phrases. Differentiable transformation groups, Stiefel manifolds, Steenrod operations.

${ }^{1}$ The research for this paper was completed when the author was supported by a Stanford University Graduate Assistantship.
} 
same integral cohomology type as $S^{2 n-3} \times S^{2 n-1}$, they are not of the same homotopy type as $S^{2 n-3} \times S^{2 n-1}[18,20]$. To detect this topological difference, one has to use, for example, Steenrod operations when $n$ is odd and Whitehead products when $n$ is even. Hence built into the study of transformation groups on $W_{n, 2}$ is the problem of utilizing homotopy information to obtain knowledge of the behavior of compact transformation groups.

In this paper we investigate the orbit structure and fixed point variety structure of smooth actions of $G(m)=\mathrm{SU}(m), \mathrm{Sp}(m), \mathrm{SO}(m)$, and $\operatorname{Spin}(m)$ on $W_{n, 2}, n$ odd, under the assumption that the identity component of a principal isotropy subgroup is again conjugate to $G\left(r_{0}\right), r_{0} \leqslant m$.

After examining properties of the regular linear models in $\S 1$, we shall give a summary of the main results of this paper in $\$ 2$. In $\$ 3$ we give some applications of the results in $\S 2$. In $\$ 4$ we give an outline of the proof of the main theorem through a detailed proof for the case $G=\operatorname{Sp}(m)$. Lastly, in $\$ \S 5$ and 6 we discuss the proofs of the cases of $\mathrm{SU}(m), \mathrm{SO}(m)$, and $\operatorname{Spin}(m)$.

The results in this paper constitute a portion of the author's doctoral dissertation written under Professor W. Y. Hsiang. The author wishes to express his gratitude to Professor Hsiang for his constant encouragement and invaluable advice and to Professor Hans Samelson for numerous discussions and general guidance.

1. The linear models. In this section we describe the characteristics of the regular linear models on $W_{n, 2}$.

We begin with some notation and conventions. We regard points in $W_{n, 2}$ as $(n \times 2)$-matrices whose columns are orthonormal to each other. Let $\mu_{m}, \nu_{m}, \rho_{m}$ denote respectively the usual representations of $\mathrm{SU}(m), \mathrm{Sp}(m), \mathrm{SO}(m)$ on $\mathbf{C}^{m}, \mathbf{H}^{m}$, $\mathbf{R}^{m}$. A smaller letter $c$ will denote complexification.

Denote by $G(m)$ any one of $\mathrm{SU}(m), \mathrm{Sp}(m), \mathrm{SO}(m)$.

Let $j$ be a nonnegative integer. By $G(m-j)$ we denote the subgroup of $G(m)$ given by the embedding $\left(\begin{array}{c}G(m-j) 0 \\ 0\end{array} I_{i}\right)$. Let $F^{(j)}=F(G(m-j), X)$ be the fixed point set of the action restricted to $G(m-j)$. For convenience we shall choose specific maximal tori in $G(m-j)$. Unless otherwise stated we follow [1] in these choices (see pp. 82-89) and use his notation. If $T$ is the chosen maximal torus in $G(m)$, then $T^{(j)}$ will denote the chosen maximal torus in $G(m-j)$. All roots and weights of representations will be defined with respect to these maximal tori and their Lie algebras. It is necessary to regard weights as elements of $H^{2}\left(B_{T} ; Q\right)$ in the canonical way. The notation we use closely follows that in $[4,6]$.

We also need maximal 2-tori. Again for $G(m)$ we choose specific maximal 2-tori $T_{2}$ and think of 2-weights as elements in $H^{1}\left(B_{T_{2}} ; \mathbf{Z} / 2\right)$. For this we follow [3, 6].

If $X$ is a $G$-space and $R$ a commutative ring, the equivariant cohomology algebra of $X$ is $H^{*}\left(X_{G} ; R\right)$, where $X_{G}=E_{G} \times_{G} X$ (see [5, pp. 52-55]). $H^{*}\left(X_{G} ; R\right)$ is a module over $R_{G}=H^{*}\left(B_{G} ; R\right)$ via the projection map $p^{*}: H^{*}\left(B_{G} ; R\right) \rightarrow H^{*}\left(X_{G} ; R\right)$. Because we are concerned with $W_{n, 2}$, it does not matter whether we use singular, Čech, Alexander-Spanier, or sheaf cohomology.

The regular linear models are actions defined by the complex representations $k\left[\mu_{m}\right], k\left[c \nu_{m}\right]$ or $k\left[c \rho_{m}\right]$ up to trivial representations. As an example, the regular 
linear models corresponding to $k \mu_{m}$ would be obtained as follows. Embed $k$ copies of $A \in \mathrm{SU}(m)$ in $\mathrm{SU}(n)$ along the diagonal to get

$$
\left(\begin{array}{llll}
A & & & \\
& \ddots & & \\
& & A & \\
& & & I
\end{array}\right) .
$$

Let $\mathrm{SU}(m)$ act on $W_{n, 2}=\mathrm{SU}(n) / \mathrm{SU}(n-2)$ by matrix multiplication (on the left) on points, which are $(n \times 2)$ matrices. This is the same as composing $k \mu_{m} \oplus$ (trivial) on the left with the usual action of $\mathrm{SU}(n)$ on $\mathrm{SU}(n) / \mathrm{SU}(n-2)$ via left translations. Similarly, we define the regular linear models of $\operatorname{Sp}(m)$ and $\mathrm{SO}(m)$ using the usual embeddings $c: \mathrm{Sp}(m) \subseteq \mathrm{SU}(2 m), c: \mathrm{SO}(m) \subseteq \mathrm{SU}(m)$.

We make the following observations.

1. For $\mathrm{SU}(m)$, necessarily $m \leqslant n$; if $m=n$ we get the usual transitive action on $W_{n, 2}$. For $\operatorname{Sp}(m)$, we must have $m \leqslant[n / 2]=$ the greatest integer less than or equal to $n / 2$. For $\mathrm{SO}(m)$, we must have $m \leqslant n$.

2. Orbit structure. We regard $G(m)$ as acting on $\mathbf{C}^{n} \times \mathbf{C}^{n}$ with $W_{n, 2}$ as an invariant submanifold. It is easily seen that the isotropy subgroups are all connected and conjugate to $G\left(m_{x}\right)$, so that the orbits are respectively complex, quaternionic, and real Stiefel manifolds. The principal isotropy types are respectively $(\mathrm{SU}(m-2 k))$, $(\mathrm{Sp}(m-2 k))$, and $(\mathrm{SO}(m-4 k))$ for $\mathrm{SU}(m)$ actions modelled after $k \mu_{m}+$ (trivial), $\mathrm{Sp}(m)$ actions modelled after $k c \nu_{m}+($ trivial), and $\mathrm{SO}(m)$ actions modelled after $k c \rho_{m}+$ (trivial).

3. The fixed point sets of these actions, if nonempty, are again complex Stiefel manifolds. Recall that the Stiefel manifold $W_{q, 2}$ has the same $\mathbf{Z} / 2$ cohomology as the product $S^{2 q-3} \times S^{2 q-1}$. Let $f_{1}$ and $f_{2}$ denote the generators of the exterior algebra $H^{*}\left(W_{q, 2} ; \mathbf{Z} / 2\right)$. Then $\mathrm{Sq}^{2} f_{1}=f_{2}$ if $q$ is odd and $\mathrm{Sq}^{2} f_{1}=0$ if $q$ is even.

For an $\mathrm{SU}(m)$ action modelled after $k \mu_{m}+$ (trivial), the fixed point set $F$ is diffeomorphic to $W_{n-k m, 2}$. Let us say that $F$ is $\mathrm{Sq}^{2}$ linked if $\mathrm{Sq}^{2} f_{1}=f_{2}$ and that $F$ is $\mathrm{Sq}^{2}$ unlinked if $\mathrm{Sq}^{2} f_{1}=0$. When $n$ is odd, then $F$ is $\mathrm{Sq}^{2}$ linked if $k$ or $m$ is even and $F$ is $\mathrm{Sq}^{2}$ unlinked if both $k$ and $m$ are odd. In fact if $F^{(j)}=F(\mathrm{SU}(m-j), X), j \geqslant 0$, and $n$ is odd, then $F^{(j)}$ is $\mathrm{Sq}^{2}$ linked whenever $m-j$ or $k$ is even and is $\mathrm{Sq}^{2}$ unlinked if both $m-j$ and $k$ are odd. Thus for $n$ odd the sequence $\left\{F^{(j)}\right\}$ is either alternately $\mathrm{Sq}^{2}$ linked (when $k$ is odd) or completely $\mathrm{Sq}^{2}$ linked (when $k$ is even). For an $\mathrm{Sp}(m)$ action $F$ is always $\mathrm{Sq}^{2}$ linked if $n$ is odd and is never $\mathrm{Sq}^{2}$ linked when $n$ is even.

The cohomological structure of the spaces $\left\{F^{(j)}=F(\mathrm{SO}(m-j), X)\right\}$ of an $\mathrm{SO}(m)$ action modelled after $k c \rho_{m}+$ (trivial) is the same as that of an $\mathrm{SU}(m)$ action modelled after $k \mu_{m}+$ (trivial).

4. Suppose $F^{(1)}=F\left(G(m-1), W_{n, 2}\right)$, where $G(m-1)$ is included in $G(m)$ as follows: $\left(\begin{array}{c}G(m-1) \\ 0\end{array}\right)$. There is an inclusion map $i: F \subseteq F^{(1)}$; we may compute $i^{*}$ : $\tilde{H}^{*}\left(F^{(1)} ; \mathbf{Z} / 2\right) \rightarrow \tilde{H}^{*}(F ; \mathbf{Z} / 2)$. Since $F^{(1)}$ and $F$ are both Stiefel manifolds and $F^{(1)}$ is $W_{n-k m+k, 2}($ for $G(m)=\mathrm{SU}(m)$ or $\mathrm{SO}(m))$ or $W_{n-2 k m+2 k, 2}($ for $G(m)=\mathrm{Sp}(m))$, it is well known that $i^{*}$ is zero unless $\operatorname{dim} F^{(1)}-\operatorname{dim} F=4$. This condition is equivalent to $k=1$ and $G(m)=\mathrm{SU}(m)$ or $\mathrm{SO}(m)$; it is never satisfied in the case of 
$\operatorname{Sp}(m)$. When $k=1, i^{*}$ is given by $i^{*}\left(f_{1}^{\prime}\right)=f_{2}$ and $i^{*}\left(f_{2}^{\prime}\right)=0$, where $H^{*}\left(F^{(1)} ; \mathbf{Z} / 2\right)$ $\approx \Lambda_{\mathbf{Z} / 2}\left(f_{1}^{\prime}, f_{2}^{\prime}\right)$ and $H^{*}(F ; \mathbf{Z} / 2) \approx \Lambda_{\mathbf{Z} / 2}\left(f_{1}, f_{2}\right)$.

5. We continue to assume $F \neq \varnothing$. Then the reduced geometrical weight system (see [14, especially Chapters IV and V]) is $\Omega_{T}^{\prime}\left(W_{n, 2}\right)=\left\{ \pm \theta_{i} ; 2 k\right\}$ for $\mathrm{SU}(m)$ and $\left\{ \pm \theta_{i}, 4 k\right\}$ for $\operatorname{Sp}(m)$. Here $\pm \theta_{i}$ are the weights of $\left[\mu_{m}\right]_{\mathbf{R}}$ or $\left[c \nu_{m}\right]_{\mathbf{R}}$, and the corresponding algebraic multiplicities are equal to $\frac{1}{2}\left(\operatorname{dim} F_{i}^{(1)}-\operatorname{dim} F\right), F_{i}^{(1)}=$ $F\left(\theta_{i}^{\perp}, W_{n, 2}\right) .\left(\theta_{i}^{\perp}\right.$ denotes the corank 1 subtorus of $T$ corresponding to $\theta_{i}(x)=0$.)

For $\mathrm{SO}(m)$, the reduced geometrical 2-weights system (see p. 65 of [14]) $\Omega_{2}^{\prime}(X, F)$ $=\left\{t_{i} ; 4 k\right\}$, where the algebraic multiplicities are $\operatorname{dim} F_{i}^{(1)}-\operatorname{dim} F, F_{i}^{(1)}=$ $F\left(t_{i}^{\perp}, W_{n, 2}\right) \cdot\left(t_{i}^{\perp}\right.$ denotes $\left.\operatorname{Ker} t_{i}.\right)$

6. Suppose $F$ is nonempty. Then for $G=\mathrm{SU}(m)$ or $\mathrm{Sp}(m)$ the equivariant cohomology of $W_{n, 2}=X$ is $H_{G}^{*}\left(W_{n, 2} ; \mathbf{Z}\right) \simeq \Lambda_{R_{G}}(\tilde{x}, \tilde{y})$, an exterior algebra over $R_{G}=$ $H^{*}\left(B_{G} ; \mathbf{Z}\right)$. Upon reduction $\bmod 2, \mathrm{Sq}^{2} \tilde{x}=\tilde{y}$ holds.

For $\mathrm{SO}(m)$ actions it is more convenient to consider $H_{G}^{*}(X ; \mathbf{Z} / 2)$. Here we need $n$ to be odd. Let $x, y$ denote the generators of $H^{*}(X ; \mathbf{Z} / 2)$. Then $\mathrm{Sq}^{2} x=y$. It is easily seen that $H_{G}^{*}(X ; \mathbf{Z} / 2)$ is isomorphic to $\Lambda_{R_{G}}(\tilde{x}, \tilde{y})$, where $R_{G}=H^{*}\left(B_{G} ; \mathbf{Z} / 2\right)$ and $\mathrm{Sq}^{2} \tilde{x}=\tilde{y}$.

2. Statement of results. In the following we shall let $\left(H^{0}\right)$ denote the conjugacy class of the identity component of a principal isotropy subgroup of the given $G$-action and shall refer to it as the connected principal isotropy type of the action.

The main result of this paper is the following.

MAIN TheOREM. Let $G(m)$ act smoothly on $X=W_{n, 2}, n$ odd, such that $\left(H^{0}\right)=$ $\left(G\left(r_{0}\right)\right)$ with $r_{0} \geqslant 4,6,11$ respectively for $\mathrm{Sp}(m), \mathrm{SU}(m)$, and $\mathrm{SO}(m)$. Then in the first two cases the actions are modelled after $\frac{1}{2}\left(m-r_{0}\right) c\left[\nu_{m}\right]+($ trivial $)$ and $\frac{1}{2}\left(m-r_{0}\right) \mu_{m}$ + (trivial) respectively. In the third case many aspects of the action resemble the regular linear models, but there is an obstruction to complete resemblance. Specifically, the following are true:

1. All isotropy subgroups are conjugate to some $G\left(m_{x}\right), m_{x} \geqslant r_{0}$. Moreover, $m-r_{0}$ is even.

2. Except when $m$ is odd and $G(m)=\mathrm{SO}(m), H^{*}\left(X_{G} ; \mathbf{Z} / 2\right) \approx \Lambda_{R_{G}}(\tilde{x}, \tilde{y})$, where $\tilde{y}=\mathrm{Sq}^{2} \tilde{x}$, and $\tilde{x}$ is chosen in some specific way (indicated in the proofs of the theorem ). If $m$ is odd and $G(m)=\mathrm{SO}(m), H^{*}\left(X_{G} ; \mathbf{Z} / 2\right)$ is a free $H^{*}\left(B_{G} ; \mathbf{Z} / 2\right)$-module with basis $1, \tilde{x}, \tilde{y}, \tilde{x} \tilde{y}$ and $\mathrm{Sq}^{2} \tilde{x}=\tilde{y}$.

Assume that $F(G(m), X) \neq \varnothing$ in the following.

3. If $G \neq \mathrm{SO}(m), m$ odd, then $F(G(m), X)=F(T, X)$ is an integral cohomology product of two odd spheres whose dimensions differ by 2 .

The maps $j^{*}: H^{*}\left(X_{G} ; \mathbf{Z} / 2\right) \rightarrow H^{*}\left(F_{G} ; \mathbf{Z} / 2\right)$ and $H^{*}\left(F_{G}^{(j+1)} ; \mathbf{Z} / 2\right) \rightarrow H^{*}\left(F_{G}^{(j)} ; \mathbf{Z} / 2\right)$ are specifically known. Also, the structure of $H^{*}(F ; \mathbf{Z} / 2)$ over the $\bmod 2$ Steenrod algebra is the same as that in the case of the regular linear models.

4. $\mathrm{Sq}^{1} \tilde{y}=0$ iff $m-r_{0} \equiv 0$ (4) for $G=\mathrm{SO}(m)$.

5. If $m$ is odd and $G=\mathrm{SO}(m)$, then $\mathrm{Sq}^{1} \tilde{y}=0$ iff $F$ is a $\mathrm{Z} / 2$-cohomology product of two odd spheres and $H^{*}\left(X_{G} ; \mathbf{Z} / 2\right)$ is an exterior algebra on $\tilde{x}, \tilde{y}$. The dimensions of the two odd spheres then differ by 2 . 
We mention here that results similar to the above have been obtained by $\mathrm{W}$. Y. Hsiang (see pp. 142-145 of [14]). While his theorems apply to more general Stiefel manifolds, our theorem contains more detailed information about the cohomological structure of the network of submanifolds $\{F(G(m-j), X)\}$ as well as about the equivariant characteristic classes of the actions. Also, his assumptions on the geometric weight system imply our assumption on connected principal isotropy type.

3. Applications. In this section we deduct a corollary of the main theorem to the effect that generically speaking a classical simple Lie group $G(m)$ acts smoothly on $W_{n, 2}, n$ odd, only if $W_{n, 2}$ admits a linear model of $G(m)$.

THEOREM 1. Let $\mathrm{SU}(m)$ or $\mathrm{Sp}(m)$ act smoothly and nontrivially on $W_{n, 2}, n$ odd. Then subject to the dimension restriction $n \geqslant 9(n \geqslant 8)$ for $\mathrm{SU}(m)(\mathrm{Sp}(m))$, we have $m \leqslant n(m \leqslant n / 2)$. In the case of $\mathrm{SU}(m)$, if $n=m>31$ and the action is effective, then it is equivalent to the standard transitive action on $W_{n, 2}$.

Proof. We consider each group separately.

(1) The $\mathrm{SU}(m)$ case. Let $(H)$ denote the principal isotropy type of the action. Then $\operatorname{dim} G-\operatorname{dim} H \leqslant \operatorname{dim} W_{n, 2}$ translates into $\operatorname{dim} G-\operatorname{dim} H \leqslant 4 n-4 \leqslant$ $4(m-1)-4$ if we assume $m>n$. It follows that $\operatorname{dim} G-\operatorname{dim} H \leqslant 4 m-8 \leqslant$ $\frac{1}{2}(m-1)^{2}$ if $m \geqslant 8$. This last condition holds since $m>n \geqslant 9$ by assumption. By Theorem 1.19 of [11, I], we conclude that $H^{0} \supseteq H_{1}$, where $H_{1}$ is a normal subgroup of $H^{0}$ conjugate to $\mathrm{SU}\left(r_{0}\right)$, with $r_{0}>m / 2$. By Theorem 1 in $\$ 3$ of [15], the possibilities for $H^{0}$ are $\mu_{r_{0}}\left(\mathrm{SU}\left(r_{0}\right)\right)$ or $2 \mu_{r_{0}}\left(\mathrm{SU}\left(r_{0}\right)\right)$. Because $r_{0}>m / 2$, the second case does not occur. Next we apply Theorem Al of $\$ 7$ of [15] to conclude that all isotropy subgroups have connected component conjugate to $\mu_{r_{x}}\left(\mathrm{SU}\left(r_{x}\right)\right)$ for $r_{x} \geqslant r_{0}>$ $m / 2 \geqslant 5$. Because of Theorem $2(\S 4)$, we see that all isotropy subgroups are in fact conjugate to $\mu_{r_{x}}\left(\mathrm{SU}\left(r_{x}\right)\right)$. By the main theorem, $m-r_{0} \equiv 0(\bmod 2)$. Observe that

$$
\operatorname{dim} \mathrm{SU}(m) / \mathrm{SU}(m-2)=4 m-4>4 n-4,
$$

a contradiction. Hence $m \leqslant n$.

If $m=n$, the above argument shows that $m-r_{0} \equiv 0$ (2). Hence $r_{0}=m-2$ or $m$. The latter case corresponds to the trivial action and the former case to a transitive action. We conclude the proof of the $\mathrm{SU}(m)$ case by quoting Theorem 4-2 in [17].

(2) The $\mathrm{Sp}(m)$ case. Assume that $m>n / 2$. By Proposition 4 of [25] the possibilities for the connected principal isotropy type of the given action are

(i) $T=$ any subtorus.

(ii) $\nu_{r}(\mathrm{Sp}(\mathrm{r}))$.

(iii) $k\left(\nu_{1}^{(1)}+\cdots+\nu_{1}^{(s)}\right)\left(\mathrm{Sp}(1)^{(1)} \times \cdots \times \mathrm{Sp}(1)^{(s)}\right), k=1,2,4$.

If $n \geqslant 7$,

$$
\operatorname{dim}\left(\frac{\operatorname{Sp}(m)}{T}\right) \geqslant 2 m^{2}+m-m=2 m^{2}>2 \cdot \frac{n^{2}}{4}=\frac{n^{2}}{2} \geqslant 4 n-4
$$

and

$$
\begin{aligned}
\operatorname{dim}(\operatorname{Sp}(m) / & (\operatorname{Sp}(1) \times \cdots \times \operatorname{Sp}(1))) \geqslant 2 m^{2}+m-3 m \\
& =2 m^{2}-2 m=2 m(m-1)>2 \cdot \frac{n}{2} \cdot \frac{n}{2}=\frac{n^{2}}{2} \geqslant 4 n-4
\end{aligned}
$$


Hence only possibility (ii) occurs if $n \geqslant 7$. By Theorem $\mathrm{Cl}$ of $\S 8$ of [15], all connected isotropy subgroups are of the type $\nu_{r_{x}}\left(\mathrm{Sp}\left(r_{x}\right)\right)$ for $r_{x} \geqslant r_{0}$. Since $\operatorname{dim}\left(\operatorname{Sp}(m) / \operatorname{Sp}\left(r_{0}\right)\right) \leqslant$ $4 n-4$, we have $2 m^{2}+m-2 r_{0}^{2}-r_{0} \leqslant 4 n-4$. So

$$
\begin{aligned}
2 r_{0}^{2}+r_{0} & \geqslant 2 m^{2}+m-4 n+4>2\left(\frac{n}{2}+1\right)^{2}+\frac{n}{2}-4 n+4 \\
& =\frac{n^{2}}{2}+\frac{n}{2}-2 n+6 \geqslant 26
\end{aligned}
$$

when $n \geqslant 8$. This implies that $r_{0}>3.25$. So by Theorem 2 (since $r_{0} \geqslant 4$ ), all isotropy subgroups are connected and $m-r_{0} \equiv 0(\bmod 2)$. Now

$$
\begin{aligned}
\operatorname{dim}\left(\frac{\operatorname{Sp}(m)}{\operatorname{Sp}(m-2)}\right) & =2+8 m-8=8 m-6 \geqslant 8 \cdot\left(\frac{n}{2}+1\right)-6 \\
& =4 n+2>4 n-4 .
\end{aligned}
$$

Hence we have obtained a contradiction.

The $\operatorname{Sp}(m)$ result is an improvement of Proposition 4 for $W_{n, 2}, n$ odd, in [25].

Let $\mathrm{SO}(m)$ act smoothly and nontrivially on $W_{n, 2}, n$ odd, and suppose $(H)$ is the principal isotropy type. Assume $m>n$, then $\operatorname{dim} G-\operatorname{dim} H \leqslant 4 n-4 \leqslant 4(m-1)$ $-4=4 m-8 \leqslant \frac{1}{4}(m-1)^{2}$ if $m \geqslant 16$. By Theorem 1.18 of [11, I], $H^{0}$ is conjugate to $\mathrm{SO}(k) \times K \subseteq \mathrm{SO}(k) \times \mathrm{SO}(m-k) \subseteq \mathrm{SO}(m)$, for $k>m / 2$ and $K \subseteq$ $\mathrm{SO}(m-k)$. By Theorem 3 of $\S 5$ of [15], $\left(H^{0}\right)$ is conjugate to $\rho_{r_{0}}\left(\mathrm{SO}\left(r_{0}\right)\right)$ with $r_{0}>m / 2$ or to $2 \rho_{r_{0}}\left(\mathrm{SO}\left(r_{0}\right)\right)$ with $r_{0}>m / 2$. Because of dimension reasons the latter cannot hold. Applying Theorem B1 of $\S 9$ of [15] we conclude that all isotropy subgroups have connected components conjugate to $\rho_{r}(\operatorname{SO}(r))$, with $r \geqslant r_{0}>m / 2$. By Theorem 2, all isotropy subgroups are connected. If $r_{0}>11$, we may apply the main theorem to conclude that $m-r_{0} \equiv 0$ (2). If $m-r_{0} \neq 2$, then $4 n-4 \geqslant$ $\operatorname{dim}(G / H) \geqslant \operatorname{dim}(\mathrm{SO}(m) / \mathrm{SO}(m-4))=4 m-5$ so that $m \leqslant n$, a contradiction. We therefore have the conclusion below:

If $\mathrm{SO}(m)$ acts smoothly and nontrivially on $W_{n, 2}, n$ odd, with $m>n \geqslant 19$, then the principal isotropy type can only be $(\mathrm{SO}(m-2))$. If the conjecture at the end of $\S 6$ is true, then all $\mathrm{SO}(m)$ actions on $X$ with $m>n \geqslant 19$ must be trivial.

REMARK. Theorem 1 says that in order that $\mathrm{SU}(m)$ or $\mathrm{Sp}(m)$ act smoothly and nontrivially on $W_{n, 2}, n$ odd and sufficiently large, it is necessary that these groups admit an $n$-dimensional complex representation. If $\mathrm{Sq}^{1} \tilde{y}=0$ and $n \geqslant 19$, the same is true of smooth $\mathrm{SO}(m)$ actions on $X$.

4. An outline of the proof of the main theorem and the specific case of $\operatorname{Sp}(m)$. In this section we give an outline of the proof of the main theorem through describing the proof for the case $G=\operatorname{Sp}(m)$. Essentially there are four main steps:

Step 1. We obtain information about the isotropy types of the given $G(m)$-action from the connected principal isotropy type.

Recall that the Stiefel manifolds are parallelizable [24] and hence $W_{*}(X)=1$, $P_{*}(X)=1$, where $W_{*}(X)$ is the total Stiefel-Whitney class of $X$ and $P_{*}(X)$ the 
total Pontrjagin class of $X$. In [15] we have the following theorems concerning local orbit structure:

Theorem A1, B1, C1 (\$§7, 8, 9 in [15]). Let $G(m)=\mathrm{SU}(m), \mathrm{Sp}(m)$, or $\mathrm{SO}(m)$, and $M$ be a compact smooth $G(m)$-manifold with $P_{1}(M)=0$. Suppose the connected principal isotropy type is $\left(H^{0}\right)=\left(\mu_{r_{0}}\left(\mathrm{SU}\left(r_{0}\right)\right)\right),\left(\nu_{r_{0}}\left(\mathrm{Sp}\left(r_{0}\right)\right)\right)$, or $\left(\rho_{r_{0}}\left(\mathrm{SO}\left(r_{0}\right)\right)\right)$ respectively with $r_{0} \geqslant 3,2$, or 5 . Then all connected isotropy groups $G_{x}^{0}$ are conjugate to $G\left(r_{x}\right)$ for $r_{x} \geqslant r_{0}$.

This is not sufficient for analysing the cohomological characteristics of the given action because $G_{x} / G_{x}^{0}$ may have all sorts of torsion. However, we have

Theorem 2. Let $G=G(m)=\mathrm{SU}(m), \mathrm{Sp}(m)$, or $\mathrm{SO}(m)$, and $X=W_{n, n-k}$, the complex Stiefel manifold of orthonormal $(n-k)$-frames in $\mathbf{C}^{n}$. If $G$ acts nontrivially and smoothly on $X$ in such $a$ way that all connected isotropy groups are conjugate to some $G\left(r_{x}\right)$ and if

(i) $k \geqslant n / 2$,

(ii) the connected principal isotropy type $\left(G\left(r_{0}\right)\right)$ has $r_{0} \geqslant 4(5$ for $\mathrm{SO}($ odd $))$ and $r_{0} \geqslant n-k+2, n-k+1,2(n-k+2)$ respectively for $\mathrm{SU}(m), \mathrm{Sp}(m)$, and $\mathrm{SO}(m)$, then in fact all isotropy subgroups are connected.

For a rough outline of the proof of this theorem we refer the reader to that of Theorem VII.2' on pp. 138-139 of [14]. It is assumed there that $F(T, X) \neq \varnothing$; however, with our assumptions and using Theorem VII.1 of [14] we may always restrict the action to an appropriate subgroup so that the fixed point set becomes nonempty. The technical part of the proof of Theorem 2 involves choosing the subgroups $K_{p}, K$ and the integer $n^{\prime}$ carefully so that the outline of the argument goes through. Because of the amount of care needed this technical part is long and not particularly enlightening. Hence we choose not to include the detailed proof here.

REMARK. For $k=n-2$, the conditions (i) and (ii) become $r_{0} \geqslant 4,4$, and 8 in the respective cases of $\mathrm{SU}(m), \mathrm{Sp}(m)$, and $\mathrm{SO}(m)$.

Step 2. We use the information about isotropy types obtained in step 1 to deduce some facts about the equivariant cohomology of $W_{n, 2}$ and of fixed point sets of subgroups of $G(m)$.

As an example, consider the case $G(m)=\mathrm{Sp}(m)$ with connected principal isotropy type $\operatorname{Sp}\left(r_{0}\right)$, where $r_{0} \geqslant 4$. In view of Theorem 2 all isotropy subgroups of the action are conjugate to $\operatorname{Sp}\left(r_{x}\right)$ for some $r_{x} \geqslant r_{0}$.

We shall also assume that $F(\operatorname{Sp}(m), X) \neq \varnothing$. This restriction is relatively mild, because if we have an action with empty fixed point set satisfying all the above assumptions then applying a theorem of Allday (Theorem VII.1 on p. 136 of [14]) we see that the original action restricted to the standardly embedded $\operatorname{Sp}(m-2)$ will have nonempty fixed point set, regular orbit structure, and nontrivial principal isotropy type. In any event we may further restrict the $\operatorname{Sp}(m)$ action to $\operatorname{Sp}(m-1)$ and still have non-empty fixed point set, regular orbit structure, and nontrivial principal isotropy type. Such restrictions are necessary, as shall be seen.

Let $X$ denote $W_{n, 2}$ from now on. 
LEMMA 1. $F=F(T, X)=F(G(m), X)$ is an integral cohomology product of two odd spheres and an orientable closed submanifold of $X$.

Proof. This lemma follows from a standard argument involving the Serre spectral sequence of the fibration $p: X_{T} \rightarrow B_{T}$, Corollary 2 on p. 46 of [14], Proposition 1 on p. 45 of [14], and Theorem IV-5 on p. 53 of [14]. (See also [9].)

LemmA 2. The maps $j^{*}: H_{T}^{*}(X ; \mathbf{Z} / 2) \rightarrow H_{T}^{*}(F ; \mathbf{Z} / 2)$ and $k^{*}: H_{G}^{*}(X ; \mathbf{Z} / 2) \rightarrow$ $H_{G}^{*}(F ; \mathbf{Z} / 2)$ are monomorphisms. Here $j^{*}$ and $k^{*}$ are induced by inclusion.

Proof. We have a commutative diagram

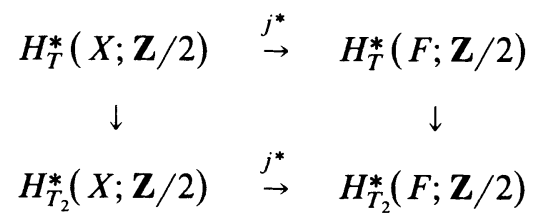

whose bottom row is a monomorphism (from a standard argument). The left-hand vertical map is injective because the mod 2 Serre spectral sequences of $X_{T} \rightarrow B_{T}$ and $X_{T_{2}} \rightarrow B_{T_{2}}$ both collapse. (Note that for this we need $\mathrm{Sq}^{2}$ connecting the generators of $H^{*}(X ; \mathbf{Z} / 2)$ in dimensions $2 n-3$ and $2 n-1$.)

LEMMA 3. $H_{G}^{*}(X ; \mathbf{Z} / 2)$ is an exterior algebra $\Lambda_{R_{G}}(\tilde{x}, \tilde{y})$, where we may choose $\tilde{x}$ to be the unique lift of the generator $x \in H^{2 n-3}(X ; \mathbf{Z} / 2)$ and $\tilde{y}=\mathrm{Sq}^{2} \tilde{x} . R_{G}$ stands for $H^{*}\left(B_{G} ; \mathbf{Z} / 2\right)$.

Proof. This is a straightforward verification using Lemma 2 and the fact that $H^{*}\left(B_{G} ; \mathbf{Z} / 2\right)$ has only even dimensional cohomology.

Likewise, we have

LEMMA 4. $H_{T}^{*}(X ; \mathbf{Z} / 2)$ is an exterior algebra $\Lambda_{R_{T}}(\tilde{x}, \tilde{y})$, where $R_{T}$ stands for $H^{*}\left(B_{T} ; \mathbf{Z} / 2\right)$. Furthermore, the map $H_{G}^{*}(X ; \mathbf{Z} / 2) \rightarrow H_{T}^{*}(X ; \mathbf{Z} / 2)$ can be given by $\tilde{x} \mapsto \tilde{x}, \tilde{y} \mapsto \tilde{y}$ (by abuse of notation) and the inclusion $H^{*}\left(B_{G} ; \mathbf{Z} / 2\right) \subseteq H^{*}\left(B_{T} ; \mathbf{Z} / 2\right)$.

Step 3. Using the topological splitting principle in [10], we set up an equation with variables in $H^{*}\left(B_{T} ; \mathbf{Z} / 2\right)$ and operator equal to $\mathrm{Sq}^{2}$.

Again, we illustrate with $G=\mathrm{Sp}(m)$.

Since $F(T, X)=F(G, X)=F$, there is a commutative diagram

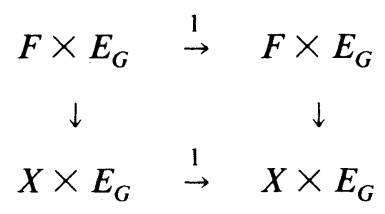


where spaces in the left column are regarded as $T$-spaces while those in the right column are regarded as $G$-spaces. All maps are equivariant and induce a commutative diagram

$$
\begin{array}{ccc}
F \times B_{T} & \stackrel{i_{1}}{\rightarrow} & F \times B_{G} \\
j \downarrow & & \downarrow k \\
X_{T} & \stackrel{i_{2}}{\rightarrow} & X_{G} .
\end{array}
$$

In cohomology, we get a commutative diagram

$$
\begin{array}{lll}
\Lambda_{R_{G}}(\tilde{x}, \tilde{y}) \simeq H_{G}^{*}(X ; \mathbf{Z} / 2) & \stackrel{i_{2}^{*}}{\rightarrow} & H_{T}^{*}(X ; \mathbf{Z} / 2) \simeq \Lambda_{R_{T}}(\tilde{x}, \tilde{y}) \\
\Lambda_{R_{G}}\left(f_{1}, f_{2}\right) \simeq H_{G}^{*}(F ; \mathbf{Z} / 2) & \stackrel{i_{1}^{*}}{\rightarrow} & H_{T}^{*}\left(F ; \stackrel{\mid}{\mathbf{Z} / 2)} \simeq \Lambda_{R_{T}}\left(f_{1}, f_{2}\right) .\right.
\end{array}
$$

Let $j^{*}(\tilde{x})=a f_{1}+b f_{2}=j^{*} i_{2}(\tilde{x})=i_{1}^{*} k^{*}(\tilde{x})=i_{1}^{*}\left(a^{\prime} f_{1}+b^{\prime} f_{2}\right)=\left(i_{1}^{*} a^{\prime}\right) f_{1}+\left(i_{1}^{*} b^{\prime}\right) f_{2}$. We conclude that $a, b$ are actually symmetric polynomials in $\theta_{1}^{2}, \ldots, \theta_{m}^{2}$, where $H^{*}\left(B_{G} ; \mathbf{Z} / 2\right) \subseteq \mathbf{Z} / 2\left[\theta_{1}, \ldots, \theta_{m}\right]$. (For $G=\mathrm{SU}(m), a, b$ are symmetric polynomials in $\theta_{1}, \ldots, \theta_{m}$.)

Let us now assume for the rest of this section that $\operatorname{dim} f_{1}=p \leqslant \operatorname{dim} f_{2}=q$. $\mathrm{Sq}^{2} j^{*}(\tilde{x})=j^{*}\left(\mathrm{Sq}^{2} \tilde{x}\right)=j^{*}(\tilde{y})=\left(\mathrm{Sq}^{2} a\right) f_{1}+a \mathrm{Sq}^{2} f_{1}+\left(\mathrm{Sq}^{2} b\right) f_{2}$ because $\mathrm{Sq}^{1} a$, $\mathrm{Sq}^{1} b$ and $\mathrm{Sq}^{2} f_{2}$ are odd dimensional, hence zero. Now there are two cases:

(i) $\mathrm{Sq}^{2} f_{1}=0$ : in this case $j^{*}(\tilde{x} \tilde{y})=\left(a \mathrm{Sq}^{2} b+b \mathrm{Sq}^{2} a\right) f_{1} f_{2}$;

(ii) $\mathrm{Sq}^{2} f_{1}=f_{2}: j^{*}(\tilde{x} \tilde{y})=\left(a^{2}+a \mathrm{Sq}^{2} b+b \mathrm{Sq}^{2} a\right) f_{1} f_{2}$.

$f_{1} f_{2}$ is a $\mathbf{Z} / 2$-orientation class of $H^{*}(F ; \mathbf{Z} / 2)$, and we may consider the ideal $I_{f_{1} f_{2}}(X, F)=\left\{d \in R_{T} \mid d f_{1} f_{2} \in \operatorname{im} j^{*}\right\}$. For the definition and information about $I_{f_{1} f_{2}}(X, F)$, we refer the reader to [10] or Chapter IV, $\$ \S 2$ and 3 of [14].

Take $c_{0}+c_{1} \tilde{x}+c_{2} \tilde{y}+c_{3} \tilde{x} \tilde{y} \in H_{T}^{*}(X ; \mathbf{Z} / 2)$ mapping to $d f_{1} f_{2}$. Then

$$
c_{0}+c_{1}\left(a f_{1}+b f_{2}\right)+c_{2}\left(\left(\mathrm{Sq}^{2} a\right) f_{1}+a\left(\mathrm{Sq}^{2} f_{1}\right)+\left(\mathrm{Sq}^{2} b\right) f_{2}\right)+c_{3} j^{*}(\tilde{x} \tilde{y})=d f_{1} f_{2} \text {. }
$$

Hence $c_{0}=0$ by inspection. Next we see that $d=c_{3}\left(a^{2}+a \mathrm{Sq}^{2} b+b \mathrm{Sq}^{2} a\right)$ if $\mathrm{Sq}^{2} f_{1}=f_{2}$ and $d=c_{3}\left(a \mathrm{Sq}^{2} b+b \mathrm{Sq}^{2} a\right)$ if $\mathrm{Sq}^{2} f_{1}=0$. So $I_{f_{1} f_{2}}(X, F)$ is the principal ideal in $R_{T}$ generated by $a^{2}+b \mathrm{Sq}^{2} a+a \mathrm{Sq}^{2} b$ or $a \mathrm{Sq}^{2} b+b \mathrm{Sq}^{2} a$ depending on whether $\mathrm{Sq}^{2} f_{1}=f_{2}$ or $\mathrm{Sq}^{2} f_{1}=0$.

The splitting theorem of Chang and Skjelbred (see [10] or Chapter IV, §3 of [14]) now gives use the other side of the equation.

LEMMA 5. For $G=\operatorname{Sp}(m)$, we have the following possibilities:

(i) If $\mathrm{Sq}^{2} f_{1}=0$, then $a \mathrm{Sq}^{2} b+b \mathrm{Sq}^{2} a=\bar{\sigma}_{m}^{m-r_{0}}$.

(ii) If $\mathrm{Sq}^{2} f_{1}=f_{2}$, then $a^{2}+a \mathrm{Sq}^{2} b+b \mathrm{Sq}^{2} a=\bar{\sigma}_{m}^{m-r_{0}}$.

$\bar{\sigma}_{i}$ denotes the ith elementary symmetric polynomial in $\theta_{1}^{2}, \ldots, \theta_{m}^{2}$. (We recall that $\left(\mathrm{Sp}\left(r_{0}\right)\right)$ is the principal isotropy type of the $\mathrm{Sp}(m)$ action.)

Proof. Let $F(x)$ containing $F$ be an $F^{0}$-variety with corank 1 generic isotropy subgroup. By definition, $F(x)$ is the connected component of $x$ in $F\left(T_{x}^{0}, X\right)$. By 
Skjelbred's theorem (Theorem VI-5 in [14]; see also [10]) $F(x)=F\left(T_{x}^{0}, X\right)$. Since $T_{x}^{0}$ is of corank $1, G_{x}=g \mathrm{Sp}(m-1) g^{-1}$ for some $g \in \mathrm{Sp}(m)$. Now $g \operatorname{Sp}(m-1) g^{-1}$ fixes a quaternionic direction and $T_{x}^{0}$ is a subtorus of the diagonal matrices in $\mathrm{Sp}(m)$. Hence $T_{x}^{0}$ is given by $\theta_{i} \equiv 0(\bmod 1)$ for some $i$ (in symbols: $\left.\theta_{i}^{\perp}=T_{x}^{0}\right)$. On the other hand, each subtorus $\theta_{i}^{\perp}$ gives rise to an $F^{0}$-variety containing $F$ with corank 1 generic isotropy subgroup.

So for the $T$-action on $X$,

$$
I_{f_{1} f_{2}}(X, F)=\left\{d \in H^{*}\left(B_{T} ; \mathbf{Q}\right) \mid d f_{1} f_{2} \in \operatorname{im}\left[H_{T}^{*}(X ; \mathbf{Q}) \rightarrow H_{T}^{*}(F ; \mathbf{Q})\right]\right\}
$$

is the principal ideal generated by $\prod_{i=1}^{n} \theta_{i}{ }^{d_{i}}$. (Recall that $F$ is an integral cohomology product of two odd spheres.) Here $d_{i}=m_{i} / 2, m_{i}=\operatorname{dim} F\left(\theta_{i}^{\perp}, X\right)-\operatorname{dim} F$. To determine $d_{i}$, we look at a slice at a fixed point $x$. The slice representation $\phi_{x}$ is $\left(m-r_{0}\right)\left[\nu_{m}\right]_{\mathbf{R}}+$ trivial representations in view of the orbit structure of the $G$-action. A corank $1 F^{0}$-variety of the type under consideration in $\left(m-r_{0}\right)\left[\nu_{m}\right]_{\mathbf{R}}$ has dimension $4\left(m-r_{0}\right)+\operatorname{dim} F$. Hence $d_{i}=m_{i} / 2=2\left(m-r_{0}\right)$.

Next we notice that $\left(\prod_{i=1}^{m} \theta_{i}^{2}\right)^{m-r_{0}}$ is actually integral and so $\left\{d \in H^{*}\left(B_{T} ; \mathbf{Z}\right) \mid\right.$ $\left.d f_{1} f_{2} \in \operatorname{im}\left[H_{T}^{*}(X ; \mathbf{Z}) \rightarrow H_{T}^{*}(F ; \mathbf{Z})\right]\right\}$ is the principal ideal in $H^{*}\left(B_{T} ; \mathbf{Z}\right)$ generated by $\left(\prod_{i=1}^{n} \theta_{i}\right)^{m-r_{0}}$. Finally, there is a corresponding statement using $\mathbf{Z} / 2$ coefficients.

Combining this with the calculations before the statement of Lemma 5 , we see that Lemma 5 is completely proved.

Step 4. The explicit solution of the equation derived in Step 3.

A priori, there may be many solutions to the equations (i) or (ii). However, it turns out that the global group action forces a set of local equations to be satisfied, and the action of the Weyl group gives the relationship between the solutions of the local equations and the solutions of the global equation. This yields a unique solution to the global equation.

As an example, we consider the case of $\operatorname{Sp}(m)$.

As remarked before (Step 2) without loss of generality, we may assume $F \neq \varnothing$. Let $r=m-r_{0}$. We first remark that the equation $a \mathrm{Sq}^{2} b+b \mathrm{Sq}^{2} a=\bar{\sigma}_{m}^{r}$ cannot hold. $a, b$ are polynomials in $\bar{\sigma}_{i}$, as was shown, and the only nonzero squares on $\bar{\sigma}_{i}$ are built up by $\mathrm{Sq}^{4 j}$. Hence we always have $a \mathrm{Sq}^{2} b+b \mathrm{Sq}^{2} a=0$.

Let us then analyze $a^{2}+a \mathrm{Sq}^{2} b+b \mathrm{Sq}^{2} a=\bar{\sigma}_{m}^{r}$. As was just shown, $a \mathrm{Sq}^{2}+$ $b \mathrm{Sq}^{2} a=0$. Hence $a^{2}=\overline{\boldsymbol{\sigma}}_{m}^{r}$ and $a=\overline{\boldsymbol{\sigma}}_{m}^{r / 2}$ so that $r$ is even.

Next, consider the following commutative diagram (Figure I). Admittedly, we do not need the entire diagram for $\operatorname{Sp}(m)$, but we do need it for $\mathrm{SU}(m)$. A few remarks about the diagram are in order. First, $T^{\prime}$ is a corank 1 subtorus of $T$ given by a weight and $T$ acts on $F^{\prime}=F\left(T^{\prime}, X\right)$, which by Lemma 1 is an integral cohomological product of 2 odd spheres. $F\left(T, F^{\prime}\right)=F$ and what we have just proved shows that $\mathrm{Sq}^{2}$ connects the cohomology generators of $H^{*}\left(F^{\prime} ; \mathbf{Z} / 2\right)$. By Lemma $2, j_{1}^{*}$ is a monomorphism. By Lemma $4, H_{T}^{*}\left(F^{\prime} ; \mathbf{Z} / 2\right) \approx \Lambda_{R_{T}}\left(f_{1}^{\prime}, f_{2}^{\prime}\right)$. The map $i_{F}^{*}$ is given by $f_{1} \mapsto f_{1}, f_{2} \mapsto f_{2}$ and $r^{*}: H^{*}\left(B_{T} ; \mathbf{Z} / 2\right) \rightarrow H^{*}\left(B_{T^{\prime}} ; \mathbf{Z} / 2\right)$, where $r^{*}$ sets $\theta_{m}$ equal to $0 . \bar{a}$ is therefore $a$ with $\theta_{m}$ set equal to $0 . T^{\prime}$ also acts on $X$ and $F^{\prime}=F(\operatorname{Sp}(m-1), X)$ and the bottom triangle is just the upper triangle of the action restricted to $\mathrm{Sp}(m-1)$. Hence $j_{2}^{\prime *}$ is a monomorphism. 


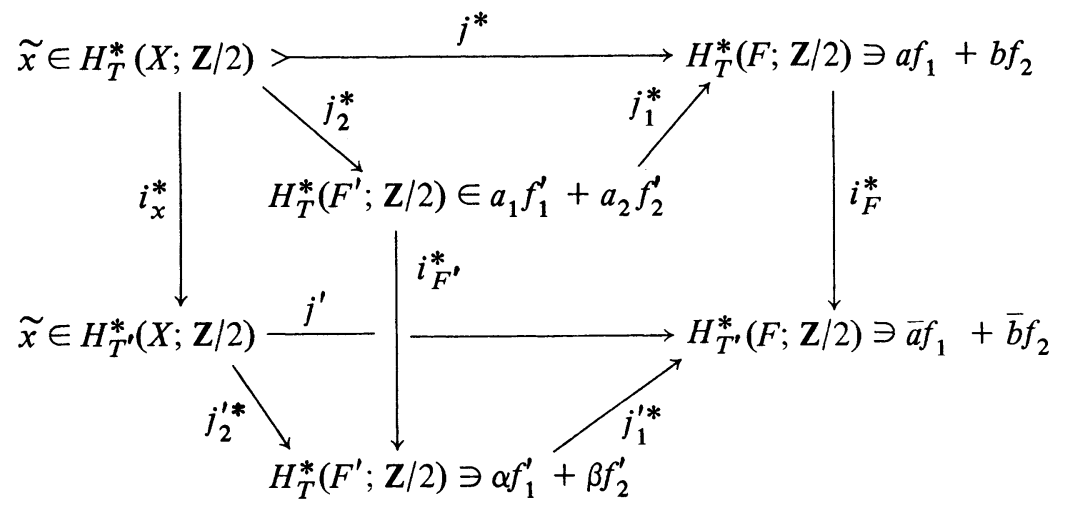

FIGURE I

Suppose now that $j_{1}^{*}\left(f_{1}^{\prime}\right)=c_{1} f_{1}+c_{2} f_{2}$. Since $F$ and $F^{\prime}$ are $\mathrm{Sq}^{2}$ linked, we have the equation $c_{1}^{2}+c_{1} \mathrm{Sq}^{2} c_{2}+c_{2} \mathrm{Sq}^{2} c_{1}=\theta_{m}^{2 r}$. This equation is obtained in exactly the same way as the equation $a^{2}+a \mathrm{Sq}^{2} b+b \mathrm{Sq}^{2} a=\bar{\sigma}_{m}^{r}$. Furthermore, let $S^{1}=$ $T / T^{\prime} . S^{1}$ acts on $F^{\prime}$ with fixed point set $F$. There is a commutative diagram

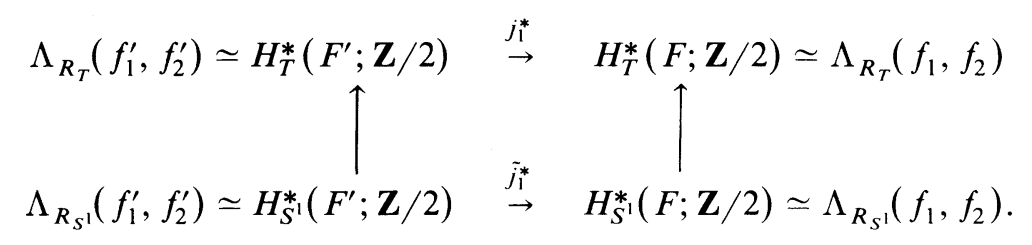

We may think of $H^{*}\left(B_{S^{1}} ; \mathbf{Z} / 2\right)$ as polynomials with $\mathbf{Z} / 2$ coefficients in $\theta_{m}$ and the vertical maps simply take $f_{i}^{\prime}$ to $f_{i}^{\prime}, f_{i}$ to $f_{i}$, and take a polynomial in $\theta_{m}$ and regards it as a polynomial in $\theta_{1}, \ldots, \theta_{m}$.

Hence we may assume that $c_{1}=e_{1} \theta_{m}^{k_{1}}, c_{2}=e_{2} \theta_{m}^{k_{2}}, e_{i}=0,1$. Clearly, $c_{1}=\theta_{m}^{r}$ because $c_{1} \neq 0$. If $e_{2} \neq 0$, we get an equation $\theta_{m}^{r} \mathrm{Sq}^{2}\left(\theta_{m}^{k_{2}}\right)+\theta_{m}^{k_{2}} \operatorname{Sq}^{2}\left(\theta_{m}^{r}\right)=0=$ $\theta_{m}^{r} k_{2} \theta_{m}^{k_{2}+1}+\theta_{m}^{k_{2}} r \theta_{m}^{r+1}$ and this forces $k_{2}+r \equiv 0$ or $k_{2} \equiv r(2)$. This contradicts $\operatorname{dim} f_{2}^{\prime}-\operatorname{dim} f_{1}^{\prime}=2$. Hence $e_{2}=0$. Now $j^{*}(\tilde{x})=a f_{1}+b f_{2}=a_{1}\left(\theta_{m}^{r} f_{1}\right)+$ $a_{2}\left(\mathrm{Sq}^{2}\left(\theta_{m}^{r}\right) f_{1}+\theta_{m}^{r} f_{2}\right)$ implies that $a=\theta_{m}^{r} a_{1}$ and $b=a_{2} \theta_{m}^{r}$. Since $a$ is a symmetric polynomial in $\theta_{i}, \sigma_{m}^{r}$ divides $a$. But $\operatorname{deg} a=2 m r$ and so $a=\sigma_{m}^{r}=\bar{\sigma}_{m}^{r / 2}$. If $a_{2} \neq 0$, then $\sigma_{m}^{r}$ divides $b$, which means $\operatorname{deg} b \geqslant 2 m r$, which is impossible. Therefore, $a_{2}=0$ and so $b=0$. Finally, $\bar{a}=0=\bar{b}$ so that $\tilde{H}^{*}\left(F^{\prime} ; \mathbf{Z} / 2\right) \rightarrow \tilde{H}^{*}(F ; \mathbf{Z} / 2)$ is zero.

At this point one observes that the main theorem for $G=\operatorname{Sp}(m)$ has been completely proved.

5. The case of $\mathrm{SU}(m)$. The discussion of the case $G=\mathrm{SU}(m)$ essentially parallels that of the case of $\operatorname{Sp}(m)$. The main difference is that Step 4 becomes more complicated.

First, we note that Lemmas 1 through 5 hold for $G=\mathrm{SU}(m)$, the only necessary change being that in Lemma 5 , one should have the elementary symmetric polynomials in $\theta_{1}, \ldots, \theta_{m}$ instead. 
Next, it should be remarked that for $\mathrm{SU}(m)$ actions, we assume that $\left(H^{0}\right)=$ $\left(\mathrm{SU}\left(r_{0}\right)\right)$, with $r_{0} \geqslant 6$, or else we assume that $r_{0} \geqslant 4$ and $F(\mathrm{SU}(m), X) \neq \varnothing$. (Compare the remarks before Lemma 1 in $\S 4$.)

We begin with

Lemma 6. $r$ is even. Also, if $m$ is even, $F$ is always $\mathrm{Sq}^{2}$-linked.

Proof. We need two formulas for this proof:

$$
\begin{aligned}
\mathrm{Sq}^{2}\left(\sigma_{i} \sigma_{j} A\right)= & \left(\mathrm{Sq}^{2} \sigma_{i}\right) \sigma_{j} A+\sigma_{i}\left(\mathrm{Sq}^{2} \sigma_{j}\right) A+\sigma_{i} \sigma_{j} \mathrm{Sq}^{2} A \\
= & \left(\begin{array}{c}
i-1 \\
1
\end{array}\right) \sigma_{i+1} \sigma_{j} A+\left(\begin{array}{c}
j-1 \\
1
\end{array}\right) \sigma_{i} \sigma_{j+1} A+\sigma_{i} \sigma_{j} \mathrm{Sq}^{2} A ; \\
& \operatorname{Sq}^{2 k}\left(\sigma_{m}^{j}\right)= \begin{cases}0 & \text { if } j \text { is even, }, \\
\sigma_{k} \sigma_{m}^{j} & \text { if } j \text { is odd. }\end{cases}
\end{aligned}
$$

Formula (ii) may be proved by induction.

First we show that $a^{2}+a \mathrm{Sq}^{2} b+b \mathrm{Sq}^{2} a=\sigma_{m}^{r}$ has a solution only if $r$ is even. Suppose $a$ does not contain a term of the form $\sigma_{m}^{k}$, then $\sigma_{m}^{r}$ must come from the term $b \mathrm{Sq}^{2} a$. Take a term in $a$ of the form $\sigma_{i} \sigma_{j} A$, where $i \neq m, j \neq m$. Then formula (i) shows that $\mathrm{Sq}^{2}\left(\sigma_{i} \sigma_{j} A\right)$ will not contribute a $\sigma_{m}^{k^{\prime}}$ term. It remains to consider a term in $a$ of the form $\sigma_{m}^{k} \sigma_{i}, i \neq m$. Now $\mathrm{Sq}^{2}\left(\sigma_{m}^{k} \sigma_{i}\right)=\left(\begin{array}{c}i-1 \\ 1\end{array}\right) \sigma_{i+1} \sigma_{m}^{k}+\sigma_{i} \mathrm{Sq}^{2}\left(\sigma_{m}^{k}\right)$. If this gives a term $\sigma_{m}^{k^{\prime}}$, then $i=m-1$ and $\left(\begin{array}{c}m-2 \\ 1\end{array}\right)$ should be $1 \bmod 2$. Hence $m$ must be odd. But $2 \operatorname{dim} a=2 m r$ and $\operatorname{dim} a$ is even. So, since $m$ is odd, $r$ must be even.

Next suppose $a$ contains a term of the form $\sigma_{m}^{k}$. Then clearly $r=2 k$.

We now study the equation $a \mathrm{Sq}^{2} b+b \mathrm{Sq}^{2} a=\sigma_{m}^{r}$ and show that a solution exists only if $m$ is odd. Suppose $\sigma_{m}^{r}$ comes from the first term. Then $\mathrm{Sq}^{2} b$ must contribute a term of the form $\sigma_{m}^{k^{\prime}}$. By what has just been shown, $b$ contains a term $\sigma_{m}^{k^{\prime}} \sigma_{m-1}$ and $m$ is odd. Similarly, if $\sigma_{m}^{r}$ comes from $b \mathrm{Sq}^{2} a$, then $a$ contains a term $\sigma_{m}^{k^{\prime}} \sigma_{m-1}$ and $m$ must be odd.

We now use the notation of the commutative diagram in the proof of the $\operatorname{Sp}(m)$ case. Let us assume $r$ is odd. Then $a \mathrm{Sq}^{2} b+b \mathrm{Sq}^{2} a=\sigma_{m}^{r}$ must hold. Hence $m$ is odd. But for the action restricted to $\mathrm{SU}(m-1)$, we either have $\alpha^{2}+\alpha \mathrm{Sq}^{2} \beta+$ $\beta \mathrm{Sq}^{2} \alpha=\hat{\alpha}_{m-1}^{r}$ or $\alpha \mathrm{Sq}^{2} \beta+\beta \mathrm{Sq}^{2} \alpha=\hat{\sigma}_{m-1}^{r}$, where $\hat{\sigma}_{m-1}$ is the $(m-1)$ st elementary symmetric polynomial in $\theta_{1}, \ldots, \theta_{m-1}$. Since $r$ is odd, the first equation cannot hold. Since $m-1$ is now even, the second equation cannot hold.

At this stage, we need to separate the discussion into cases.

Case $1 . F$ and $F^{\prime}$ are $\mathrm{Sq}^{2}$-linked. We refer the reader to Figure $\mathrm{I}$, and the subsequent remarks. Let $j_{1}^{*}\left(f_{1}^{\prime}\right)=u_{1} f_{1}+u_{2} f_{2}$. Since $\mathrm{Sq}^{2} f_{1}^{\prime}=f_{2}^{\prime}$ by assumption, we have $u_{1}^{2}+u_{1} \mathrm{Sq}^{2} u_{2}+u_{2} \mathrm{Sq}^{2} u_{1}=\theta_{m}^{r}$. Write $r=2 k$. In view of lemma let $u_{1}=e_{1} \theta_{m}^{k_{1}}$ and $u_{2}=e_{2} \theta_{m}^{k_{2}}$. Since $u_{1} \neq 0, u_{1}=\theta_{m}^{k}$. Substituting into the equation we get

$$
0=\theta_{m}^{k} \mathrm{Sq}^{2}\left(e_{2} \theta_{m}^{k_{2}}\right)+e_{2} \theta_{m}^{k_{2}} \mathrm{Sq}^{2}\left(\theta_{m}^{k}\right)=e_{2}\left(\theta_{m}^{k} k_{2} \theta_{m}^{k_{2}+1}+\theta_{m}^{k_{2}} k \theta_{m}^{k+1}\right) \text {. }
$$


If $e_{2} \neq 0$, then $k \equiv k_{2}(2)$. Adding the degrees, however, yields $k+k_{2}+1=2 k$, contradicting $k \equiv k_{2}(2)$. Hence $e_{2}=0$.

Now $j_{1}^{*}\left(f_{1}^{\prime}\right)=\theta_{m}^{k} f_{1}$ and $j_{1}^{*}\left(f_{2}^{\prime}\right)=k \theta_{m}^{k+1} f_{1}+\theta_{m}^{k} f_{2}$. It follows that

$$
j^{*}(\tilde{x})=j_{1}^{*}\left(a_{1} f_{1}^{\prime}+a_{2} f_{2}^{\prime}\right)=\left(a_{1} \theta_{m}^{k}+a_{2} k \theta_{m}^{k+1}\right) f_{1}+\left(a_{2} \theta_{m}^{k}\right) f_{2} .
$$

Since $a \neq 0, \theta_{m}^{k} \mid a$ implies that $\sigma_{m}^{k} \mid a$ ( $a$ is a symmetric polynomial), whence $a=\sigma_{m}^{k}$. Since $\operatorname{dim} a>\operatorname{dim} b$, it is clear that $b=0$ and $a_{2}=0$. Therefore, $a_{1}=\prod_{i=1}^{m-1} \theta_{i}^{k}$.

Let us now consider

$$
\begin{aligned}
\mathrm{Sq}^{4} j^{*}(x) & =\mathrm{Sq}^{4}\left(\sigma_{m}^{k} f_{1}\right)=\mathrm{Sq}^{4}\left(\sigma_{m}^{k}\right) f_{1}+\mathrm{Sq}^{2}\left(\sigma_{m}^{k}\right) f_{2} \\
& =\operatorname{Sq}^{4}\left(\sigma_{m}^{k}\right) f_{1}= \begin{cases}\sigma_{2} \sigma_{m}^{k} f_{1} & \text { if } k \text { is odd }, \\
0 & \text { if } k \text { is even. }\end{cases}
\end{aligned}
$$

Since $j^{*}$ is a monomorphism, $\mathrm{Sq}^{4} x=0$ iff $k$ is even and $\mathrm{Sq}^{4} x=\sigma_{2} x$ iff $k$ is odd.

We also see that $\tilde{H}^{*}\left(F^{\prime} ; \mathbf{Z} / 2\right) \rightarrow \tilde{H}^{*}(F ; \mathbf{Z} / 2)$ is the zero map. To summarize then, when $F$ and $F^{\prime}$ are both $\mathrm{Sq}^{2}$-linked, it follows that $a=\sigma_{m}^{k}$ and $b=0$. The homomorphism $\tilde{H}^{*}\left(F^{\prime} ; \mathbf{Z} / 2\right) \rightarrow \tilde{H}^{*}(F ; \mathbf{Z} / 2)$ is 0 .

Case 2: $F$ is not $\mathrm{Sq}^{2}$-linked. Similar, but more complicated, calculations show that when $F$ is not $\mathrm{Sq}^{2}$-linked, $F^{\prime}$ has to be $\mathrm{Sq}^{2}$-linked and $m$ must be odd. Also, $\operatorname{dim} f_{2}-\operatorname{dim} f_{1}=2$ and $j^{*}(\tilde{x})=\sigma_{m}^{k} f_{1}+\sigma_{m}^{k-1} \sigma_{m-1} f_{2}$. The map $\tilde{H}^{*}\left(F^{\prime} ; \mathbf{Z} / 2\right) \rightarrow$ $\tilde{H}^{*}(F ; \mathbf{Z} / 2)$ is zero if $k>1$ and is given by $f_{1}^{\prime} \mapsto f_{2}, f_{2}^{\prime} \mapsto 0$ if $k=1$.

Case 3: $F$ is $\mathrm{Sq}^{2}$-linked but $F^{\prime}$ is not $\mathrm{Sq}^{2}$-linked. In this case $m$ is even (since $F^{\prime}$ is not $\mathrm{Sq}^{2}$-linked) and by the result of Case $2, \operatorname{dim} f_{2}^{\prime}-\operatorname{dim} f_{1}^{\prime}=2$. (Recall $r_{0} \geqslant 6 !$.) By similar calculations as those in the previous cases, we see that $j^{*}(\tilde{x})=\sigma_{m}^{k} f_{1}+$ $\sigma_{m}^{k-1} \sigma_{m-1} f_{2}$. The map $\tilde{H}^{*}\left(F^{\prime} ; \mathbf{Z} / 2\right) \rightarrow \tilde{H}^{*}(F ; \mathbf{Z} / 2)$ is 0 if $k>1$ and when $k=1$, it is given by $f_{1}^{\prime} \mapsto f_{2}, f_{2}^{\prime} \mapsto 0$.

By combining the results of these three cases, it can be seen that the main theorem has been completely proved for $G=\operatorname{SU}(m)$.

6. The case of $\mathrm{SO}(m)$ and $\operatorname{Spin}(m)$. In proving the main theorem the $\mathrm{SO}(m)$ case is the most difficult case to deal with. The basic reason is that toral rank no longer distinguishes the isotropy types of an $\mathrm{SO}(\mathrm{m})$-action of regular type. Consequently, the $\mathbf{Z} / 2$-tori instead of the tori of $\operatorname{SO}(m)$ serve better to capture the characteristics of the group action. Unfortunately, a priori, fixed point sets of $\mathbf{Z} / 2$-tori need no longer be connected. (See [23].) Moreover, in using $\mathbf{Z} / 2$-tori, we lose track of the parity of the dimensions of the generators of the $\mathbf{Z} / 2$-cohomology of the fixed point varieties. All these cause difficulties in the proof of the main theorem.

Before proceeding further we remark that the case of $\operatorname{Spin}(m)$ actions can be reduced to that of $\operatorname{SO}(m)$ actions. The reason is that given a $\operatorname{Spin}(m)$ action of regular type, the geometric weight system is $k\left\{ \pm \theta_{i}\right\}$ and hence $\{ \pm 1\}$ acts trivially on $W_{n, 2}$. The action therefore factors through $\mathrm{SO}(m)$.

Let us now state the assumptions we need for this section. We assume $\mathrm{SO}(m)$ acts smoothly on $W_{n, 2}, n$ odd, with connected principal isotropy type $\left(\mathrm{SO}\left(r_{0}\right)\right)$ and $r_{0} \geqslant 8$. In view of Theorem 2, all isotropy subgroups of the action are conjugate to $\operatorname{SO}\left(r_{x}\right)$ for some $r_{x} \geqslant r_{0}$. We shall also assume that $F\left(\operatorname{SO}(m), W_{n, 2}\right) \neq \varnothing$. This assumption is 
again relatively harmless; for if we have an action with empty fixed point set and $r_{0} \geqslant 11$ satisfying all the assumptions above, we may appeal to Theorem VII.1 on $\mathrm{p}$. 136 of [14] and see that if we restrict the $G$-action to the standardly embedded $\mathrm{SO}(m-4)$, the resulting action will have principal isotropy type $\left(\mathrm{SO}\left(r_{0}\right)\right)$ with $r_{0} \geqslant 7$ and a nonempty fixed point set. Because the original action has orbit structure modelled after the regular linear model so does the restricted action. The condition $r_{0} \geqslant 7$ is needed because we shall have to further restrict the action by as many as five steps and we do not want to end up with a trivial principal isotropy type.

LEMMA 7. When $m$ is even, $F=F(\operatorname{SO}(m), X)=F\left(T_{2}, X\right)$ is an integral cohomology product of two odd spheres.

Proof. We note that when $m$ is even, then $F\left(T_{2}, X\right)=F(T, X)=F(G, X)=$ $F\left(T_{p}, X\right)$, where $T_{p}$ is a maximal $p$-torus in $G$ and $p$ is an odd prime. Then a proof along the lines of that of Lemma 1 can be constructed easily.

When $m$ is odd, $F\left(T_{2}, X\right)=F(G, X) \neq F(T, X)$ even in the regular linear models.

LEMMA 8. The maps $j^{*}: H_{T_{2}}^{*}(X ; \mathbf{Z} / 2) \rightarrow H_{T_{2}}^{*}(F ; \mathbf{Z} / 2)$ and $k^{*}: H_{G}^{*}(X ; \mathbf{Z} / 2) \rightarrow$ $H_{G}^{*}(F ; \mathbf{Z} / 2)$ are monomorphisms.

Proof. Similar to that of Lemma 2.

LeMma 9. Let $m$ be even. Suppose $\tilde{x}$ is a lift of $x \in H^{2 n-3}(X ; \mathbf{Z} / 2)$ in $H_{G}^{2 n-3}(X ; \mathbf{Z} / 2)$ such that $k^{*}(\tilde{x})$ does not involve a constant term and if $\tilde{y}=\mathrm{Sq}^{2} \tilde{x}$, then $H_{G}^{*}(X ; \mathbf{Z} / 2) \approx \Lambda_{R_{G}}(\tilde{x}, \tilde{y})$, where $R_{G}=H^{*}\left(B_{G} ; \mathbf{Z} / 2\right)$. Also, $H_{T_{2}}^{*}(X ; \mathbf{Z} / 2) \approx$ $\Lambda_{R_{T_{2}}}(\tilde{x}, \tilde{y})$, where $R_{T_{2}}=H^{*}\left(B_{T_{2}}, \mathbf{Z} / 2\right)$ and the map $H_{G}^{*}(X ; \mathbf{Z} / 2) \rightarrow H_{T_{2}}^{*}(X ; \mathbf{Z} / 2)$ is given by $\tilde{x} \rightarrow \tilde{x}, \tilde{y} \rightarrow \tilde{y}$ (by abuse of notation) and by the injection $H^{*}\left(B_{G} ; \mathbf{Z} / 2\right) \approx$ $\mathbf{Z} / 2\left[w_{2}, \ldots, w_{m}\right] \subseteq H\left(B_{T_{2}} ; \mathbf{Z} / 2\right) \approx \mathbf{Z} / 2\left[t_{1}, \ldots, t_{m}\right]$.

We next explain how we consistently choose generators for the cohomology algebras we shall encounter. There is a canonical map $\eta^{*}: H_{\mathrm{SO}(m)}^{*}(X ; \mathbf{Z} / 2) \rightarrow$ $H_{\mathrm{SO}(m-1)}^{*}(X ; \mathbf{Z} / 2)$. Choose $p \in F=F(G, X)$. We have the map

$$
i_{p}^{*}: H_{\mathrm{SO}(m)}^{*}(X ; \mathbf{Z} / 2) \stackrel{k^{*}}{\rightarrow} H_{\mathrm{SO}(m)}^{*}(F ; \mathbf{Z} / 2) \stackrel{\pi^{*}}{\rightarrow} H_{\mathrm{SO}(m)}^{*}(p ; \mathbf{Z} / 2) \approx H^{*}\left(B_{G} ; \mathbf{Z} / 2\right) .
$$

Let $\tilde{x}$ be a lift of $x \in H^{2 n-3}(X ; \mathbf{Z} / 2)$ such that $i_{p}^{*}(\tilde{x})=0$. (If $i_{p}^{*}(\tilde{x})=c$, just replace $\tilde{x}$ by $\tilde{x}-c$; it will still be a lift of $x$ since $i^{*} c$ is 0 , where $i^{*}: H_{G}^{*}(X ; \mathbf{Z} / 2) \rightarrow$ $H_{\{e\}}^{*}(X ; \mathbf{Z} / 2)$.) Let $\tilde{y}=\mathrm{Sq}^{2} \tilde{x}$. Then $1, \tilde{x}, \tilde{y}, \tilde{x} \tilde{y}$ form an $R_{G}$-base for $H_{G}^{*}(X ; \mathbf{Z} / 2)$. (That $H_{G}^{*}(X ; \mathbf{Z} / 2)$ is a free $H^{*}\left(B_{G} ; \mathbf{Z} / 2\right)$-module of rank 4 does not depend on the parity of $m$.) Denote by $\tilde{x}$ also the element $\eta^{*}(\tilde{x}) \in H_{\mathrm{SO}(m-1)}^{*}(X ; \mathbf{Z} / 2)$. We have similar elements in $H_{T_{2}}^{*}(X ; \mathbf{Z} / 2)$ and $H_{T_{2}^{\prime}}^{*}(X ; \mathbf{Z} / 2)$, all of which are lifts of $x \in$ $H^{2 n-3}(X ; \mathbf{Z} / 2)$. Under the maps $H_{\mathrm{SO}(m-1)}^{*}(X ; \mathbf{Z} / 2) \rightarrow H_{\mathrm{SO}(m-1)}^{*}\left(F^{\prime} ; \mathbf{Z} / 2\right) \rightarrow$ $H_{\mathrm{SO}(m-1)}^{*}(p ; \mathbf{Z} / 2), \quad H_{T_{2}^{\prime}}^{*}(X ; \mathbf{Z} / 2) \rightarrow H_{T_{2}^{\prime}}^{*}\left(F^{\prime} ; \mathbf{Z} / 2\right) \rightarrow H_{T_{2}^{\prime}}^{*}(p ; \mathbf{Z} / 2), \quad H_{T_{2}}^{*}(X ; \mathbf{Z} / 2) \rightarrow$ $H_{T_{2}}^{*}(F ; \mathbf{Z} / 2) \rightarrow H_{T_{2}}^{*}(p ; \mathbf{Z} / 2), \tilde{x}$ goes to 0 by naturality. In other words, under the maps $H_{\mathrm{SO}(m-1)}^{*}(X ; \mathbf{Z} / 2) \rightarrow H_{\mathrm{SO}(m-1)}^{*}\left(F^{\prime} ; \mathbf{Z} / 2\right)$ and $H_{T_{2}^{\prime}}^{*}(X ; \mathbf{Z} / 2) \rightarrow H_{T_{2}^{\prime}}^{*}\left(F^{\prime} ; \mathbf{Z} / 2\right), \tilde{x}$ 
goes to an element without constant term. All this can be summarized by the following commutative diagram:

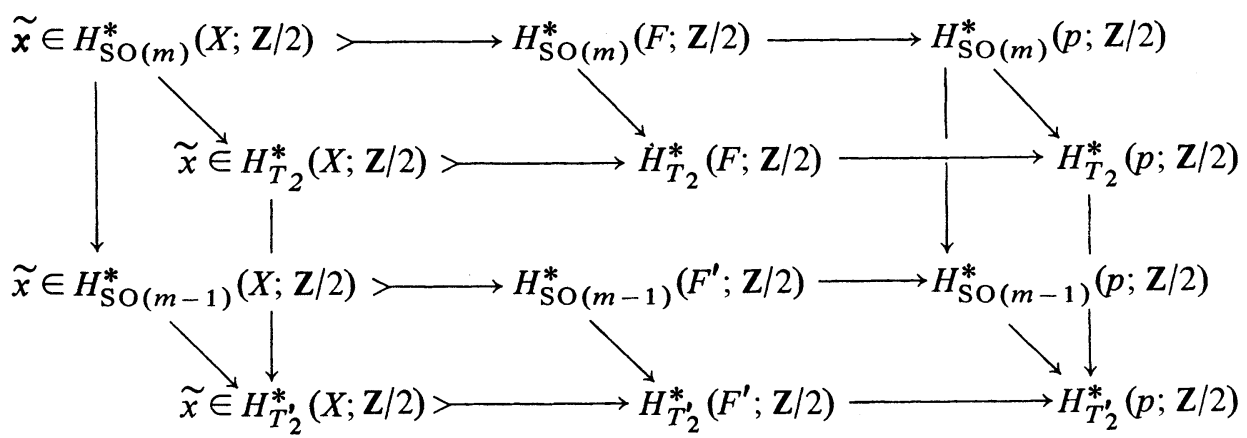

FIGURE II

(Note that there are no vertical maps in the middle of the diagram!)

The above discussion together with Lemma 9 imply that if $m$ is odd, then $H_{\mathrm{SO}(m-1)}(X ; \mathbf{Z} / 2) \approx \Lambda_{R_{\mathrm{SO}(m-1)}}(\tilde{x}, \tilde{y}), H_{T_{2}^{\prime}}^{*}(X ; \mathbf{Z} / 2) \approx \Lambda_{R_{T_{2}^{\prime}}}(\tilde{x}, \tilde{y})$. Also, the maps $H_{\mathrm{SO}(m)}^{*}(X ; \mathbf{Z} / 2) \rightarrow H_{\mathrm{SO}(m-1)}^{*}(X ; \mathbf{Z} / 2)$ and $H_{T_{2}}^{*}(X ; \mathbf{Z} / 2) \rightarrow H_{T_{2}^{\prime}}^{*}(X ; \mathbf{Z} / 2)$ are given by $\tilde{x} \rightarrow \tilde{x}, \tilde{y} \rightarrow \tilde{y}, \tilde{x} \tilde{y} \rightarrow \tilde{x} \tilde{y}$ and by the maps

$$
H^{*}(B \mathrm{SO}(m) ; \mathbf{Z} / 2) \rightarrow H^{*}(B \mathrm{SO}(m-1) ; \mathbf{Z} / 2)
$$

and

$$
H^{*}\left(B_{T_{2}} ; \mathbf{Z} / 2\right) \rightarrow H^{*}\left(B_{T_{2}^{\prime}} ; \mathbf{Z} / 2\right)
$$

REMARKs. Observe that the procedure we described above works even if $m$ is odd and if $F$ is disconnected. For the rest of this section we shall assume the above choice of generators for all cohomology algebras $H_{\mathrm{SO}(m-j)}^{*}(X ; \mathbf{Z} / 2)$ and $H_{T_{2}^{(j)}}^{*}(X ; \mathbf{Z} / 2)$, where $T_{2}^{(j)}$ is the standard corank $j$ 2-torus in $T_{2}$ obtained by setting $e_{m-j+1}=\cdots=e_{m}=1$. Finally note that if $H_{\mathrm{SO}(m)}^{*}(X ; \mathbf{Z} / 2) \approx \Lambda_{R_{\mathrm{SO}(m)}}(\tilde{x}, \tilde{y})$ then $H_{\mathrm{SO}(m-j)}^{*}(X ; \mathbf{Z} / 2) \approx \Lambda_{R_{\mathrm{SO}(m-j)}}(\tilde{x}, \tilde{y})$ and $H_{T_{2}^{(j)}}^{*}(X ; \mathbf{Z} / 2) \approx \Lambda_{R_{T_{2}(j)}}(\tilde{x} \tilde{y})$. Hence we state

LEMma 10. If $m$ is odd, then for $1 \leqslant j \leqslant\left[\frac{1}{2}\left(r_{0}-2\right)\right], H^{*}(F(\operatorname{SO}(m-2 j), X) ; \mathbf{Z} / 2)$ $\approx \Lambda_{\mathbf{Z} / 2}\left(f_{1}^{(2 j)}, f_{2}^{(2 j)}\right)$ and $H_{\mathrm{SO}(m-2 j)}(X ; \mathbf{Z} / 2) \approx \Lambda_{R_{\mathrm{SO}(m-2 j)}}(\tilde{x}, \tilde{y}), H_{T_{2}^{(2 j)}}^{*}(X ; \mathbf{Z} / 2) \approx$ $\Lambda_{R_{T_{2}(2 j)}}(\tilde{x}, \tilde{y})$.

Proof. The last assertions follow from the above remarks. Applying Proposition 1 on p. 45 of [14] we see that $S^{-1} H_{T_{2}^{(2 j)}}^{*}(X ; \mathbf{Z} / 2)$ is an exterior algebra on $\tilde{x}, \tilde{y}$ over

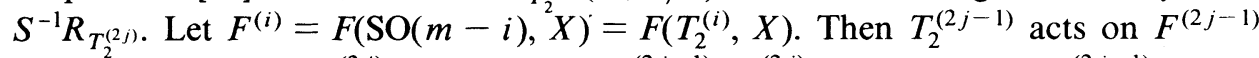
with fixed point set $F^{(2 j)}$; equivalently, $T_{2}^{(2 j-1)} / T_{2}^{(2 j)} \approx \mathbf{Z} / 2$ acts on $F^{(2 j-1)}$, which is an integral cohomology product of two odd spheres, with fixed point set $F^{(2 j)}$. The possibilities for $H^{*}\left(F^{(2 j)} ; \mathbf{Z} / 2\right)$ are given in [23] or on p. 410 of [8]. From this we see that $S^{-1} H_{T_{2}^{(2 j)}}^{*}\left(F^{(2 j)} ; \mathbf{Z} / 2\right)$ is an exterior algebra iff the first assertion of the lemma holds.

We shall now make the assumption that $F^{(j)} \sim_{\mathbf{z}_{2}} S^{p_{j}} \times S^{q_{j}}$ in what follows until p. 608. This means that for $m$ odd we just restrict the action to $\mathrm{SO}(m-1)$. 
We proceed to set up equations with variables in $H^{*}\left(B_{T_{2}} ; \mathbf{Z} / 2\right)$ involving Steenrod squares. First we take the remarks at the beginning of Step 3 in $\S 4$, replace $T$ by $T_{2}$, and note that every statement remains true. Namely, there is a commutative diagram

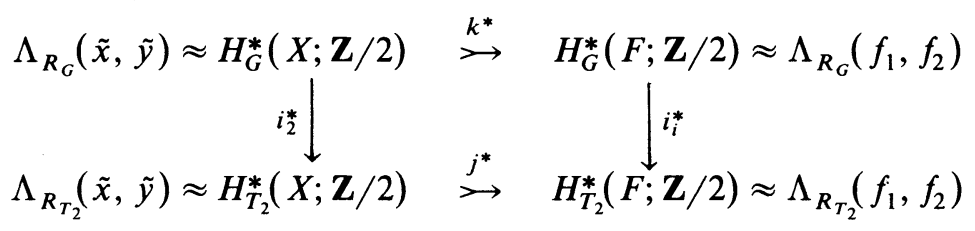

Let $j^{*}(\tilde{x})=a f_{1}+b f_{2}+c f_{1} f_{2}$, then $j^{*} i_{2}^{*}(\tilde{x})=i_{1}^{*} k^{*}(\tilde{x})=i_{1}^{*}\left(a^{\prime} f_{1}+b^{\prime} f_{2}+c^{\prime} f_{1} f_{2}\right)=$ $\left(i_{1}^{*} a^{\prime}\right) f_{1}+\left(i_{1}^{*} b^{\prime}\right) f_{2}+\left(i_{1}^{*} c^{\prime}\right) f_{1} f_{2}$. Hence we conclude as in $\$ 3$ that $a, b, c$ are symmetric polynomials in $t_{1}, \ldots, t_{m}$. Now $\operatorname{Sq}^{2}\left(j^{*} \tilde{x}\right)=j^{*}(\tilde{y})=\left(\mathrm{Sq}^{2} a\right) f_{1}+\left(\mathrm{Sq}^{1} a\right)\left(\mathrm{Sq}^{1} f_{1}\right)+$ $a \mathrm{Sq}^{2} f_{1}+\left(\mathrm{Sq}^{2} b\right) f_{2}+\left(\mathrm{Sq}^{1} b\right)\left(\mathrm{Sq}^{1} f_{2}\right)+b\left(\mathrm{Sq}^{2} f_{2}\right)+\left(\mathrm{Sq}^{2} c\right) f_{1} f_{2}$. Let $F \sim_{\mathbf{z} / 2} S^{p} \times$ $S^{q}, p \leqslant q$.

Now $\mathrm{Sq}^{1} f_{2}=0$ by considering the properties of the Bockstein homomorphism corresponding to the sequence $0 \rightarrow \mathbf{Z} / 2 \rightarrow \mathbf{Z} / 4 \rightarrow \mathbf{Z} / 2 \rightarrow 0$.

Next we consider $\operatorname{Sq}^{1} f_{1}$. If $\mathrm{Sq}^{1} f_{1} \neq 0$, then either $\mathrm{Sq}^{1} f_{1}=f_{1} f_{2}$ or $\mathrm{Sq}^{1} f_{1}=f_{2}$. In the first case, $\operatorname{dim} f_{2}=1$, and since we assumed $\operatorname{dim} f_{1} \leqslant \operatorname{dim} f_{2}$, we have $\operatorname{dim} f_{1}=1$ and so $\mathrm{Sq}^{1} f_{1}=f_{1}^{2}=f_{1} f_{2}=0$, a contradiction. In the second case, $\mathrm{Sq}^{1} f_{1}=f_{2}$ and we have two subcases.

(i) $\mathrm{Sq}^{2} f_{1}=0: j^{*}(\tilde{y})=\left(\mathrm{Sq}^{2} a\right) f_{1}+\left(\mathrm{Sq}^{1} a\right) f_{2}+\left(\mathrm{Sq}^{2} b\right) f_{2}+b \mathrm{Sq}^{2} f_{2}+\left(\mathrm{Sq}^{2} c\right) f_{1} f_{2}$. Consequently, $j^{*}(\tilde{x} \tilde{y})=\left(a \mathrm{Sq}^{1} a+a \mathrm{Sq}^{2} b+b \mathrm{Sq}^{2} a\right) f_{1} f_{2}$.

(ii) $\mathrm{Sq}^{2} f_{1}=f_{1} f_{2}$ : This cannot occur because otherwise $\operatorname{dim} f_{2}=2$ and so $\operatorname{dim} f_{1}$ $=1$ or 2 ; in either case $\mathrm{Sq}^{2} f_{1}=0$.

If $\mathrm{Sq}^{1} f_{1}=0$, we have the following cases:

(iii) $\mathrm{Sq}^{2} f_{1}=f_{2}: j^{*}(\tilde{y})=\left(\mathrm{Sq}^{2} a\right) f_{1}+a f_{2}+\left(\mathrm{Sq}^{2} b\right) f_{2}+b \mathrm{Sq}^{2} f_{2}+\left(\mathrm{Sq}^{2} c\right) f_{1} f_{2}$ and $j(\tilde{x} \tilde{y})=\left(a^{2}+a \mathrm{Sq}^{2} b+b \mathrm{Sq}^{2} a\right) f_{1} f_{2}$.

(iv) $\mathrm{Sq}^{2} f_{1}=0: j^{*}(\tilde{y})=\left(\mathrm{Sq}^{2} a\right) f_{1}+\left(\mathrm{Sq}^{2} b\right) f_{2}+b \mathrm{Sq}^{2} f_{2}+\left(\operatorname{Sq}^{2} c\right) f_{1} f_{2}$ and so $j^{*}(\tilde{x} \tilde{y})=\left(a \mathrm{Sq}^{2} b+b \mathrm{Sq}^{2} a\right) f_{1} f_{2}$.

(v) $\mathrm{Sq}^{2} f_{1}=f_{1} f_{2}$ : As in (ii), this case cannot occur.

It will turn out that case (i) is also fictitious.

$f_{1} f_{2}$ is a $\mathbf{Z} / 2$-orientation class of $H^{*}(F ; \mathbf{Z} / 2)$. So we may consider the ideal $I_{f_{1} f_{2}}(X, F)=\left\{\alpha \in R_{T_{2}} \mid \alpha f_{1} f_{2} \in \mathrm{im} j^{*}\right\}$. Exactly the same argument as in the $\operatorname{SU}(m)$ or $\mathrm{Sp}(m)$ case shows that $I_{f_{1} f_{2}}(X, F)$ is a principal ideal in $R_{T_{2}}$ generated by $a^{2}+a \mathrm{Sq}^{2} b+b \mathrm{Sq}^{2} a, a \mathrm{Sq}^{2} b+b \mathrm{Sq}^{2} a$, or $a \mathrm{Sq}^{1} a+a \mathrm{Sq}^{2} b+b \mathrm{Sq}^{2} a$ in cases (i), (iii), or (iv).

LEMMA 11. For $G=\mathrm{SO}(m)$, we have the following possibilities:

(i) If $\mathrm{Sq}^{2} f_{1}=f_{2}$, then $a^{2}+a \mathrm{Sq}^{2} b+b \mathrm{Sq}^{2} a=w_{m}^{m-r_{0}}$.

(ii) If $\mathrm{Sq}^{2} f_{1}=0$, then $a \mathrm{Sq}^{2} b+b \mathrm{Sq}^{2} a=w_{m}^{m-r_{0}}$ for $\mathrm{Sq}^{1} f_{1}=0$ and $a \mathrm{Sq}^{1} a+$ $a \mathrm{Sq}^{2} b+b \mathrm{Sq}^{2} a=w_{m}^{m-r_{0}}$ for $\mathrm{Sq}^{1} f_{1}=f_{2}$.

In the above, $w_{i}$ denotes the ith elementary symmetric polynomial in $t_{1}, \ldots, t_{m}$. We recall that $\left(\mathrm{SO}\left(r_{0}\right)\right)$ is the principal isotropy type of the G-action.

Proof. Similar to that of Lemma 5.

Because the proof of the main theorem for $\mathrm{SO}(m)$ is rather complicated and long, we give first an outline of it below. 
Stage one. Assume that $F^{(j)} \sim_{\mathrm{z} / 2} S^{p_{j}} \times S^{q_{j}}, 0 \leqslant j \leqslant r_{0}-2$, so that $H_{\mathrm{SO}(m-j)}^{*}(X ; \mathbf{Z} / 2) \approx \Lambda_{R_{\mathrm{SO}(m-j)}}(\tilde{x}, \tilde{y})$. (This is no assumption when $m$ is even and only $j=0$ is a real hypothesis when $m$ is odd.)

1. Assume $m \equiv 0$ (4). We show that $F$ is $\mathrm{Sq}^{2}$ linked (Lemma 14).

2. We prove assertion 1 in the main theorem in Lemma 15 , and hence eliminate the case $\mathrm{Sq}^{1} f_{1}=f_{2}$ in Lemma 11 .

3. Assume $m \equiv 1$ (4); we solve for $a$ and $b$ in the cases $F$ is $\mathrm{Sq}^{2}$ linked as well as $\mathrm{Sq}^{2}$ unlinked. We also show that $\mathrm{Sq}^{1} y=0$ iff 4 divides $r$.

4. Assume that $m \equiv 2$ (4). We show that $F$ has to be $\mathrm{Sq}^{2}$ linked (Lemma 16).

5. Solve for $a, b$ in the case $m \equiv 2(4)$. Hence we know $a, b$ in all cases. From all this, assertions 2 and 3 of the main theorem follow.

Stage Two (pp. 608-609). Assume that 4 divides $r$. Then we prove assertion 5 in the main theorem. As a result we can solve for $a$ and $b$ in this case from the computations in Stage one.

We proceed with Stage one of our proof.

As in $\S 3$, there is a basic commutative diagram (Figure III) that we shall refer to constantly. We make a few remarks about this diagram. We assume that $F$, $F^{\prime}=F(\mathrm{SO}(m-1), X), F^{\prime \prime}=F(\mathrm{SO}(m-2), X)=F\left(T_{2}^{\prime \prime}, X\right)$, where $T_{2}^{\prime \prime}$ is the subgroup of $T_{2}$ with $e_{m-1}=e_{m}=1$, are all $\mathbf{Z} / 2$-cohomology products of 2 spheres. Whenever these equal $F(\mathrm{SO}(m-j), X)$ with $m-j$ even, by Lemma 7 , they are Z-cohomology products of 2 odd spheres. By Lemma $8, j^{*}$ and $j_{2}^{\prime *}$ are injective.

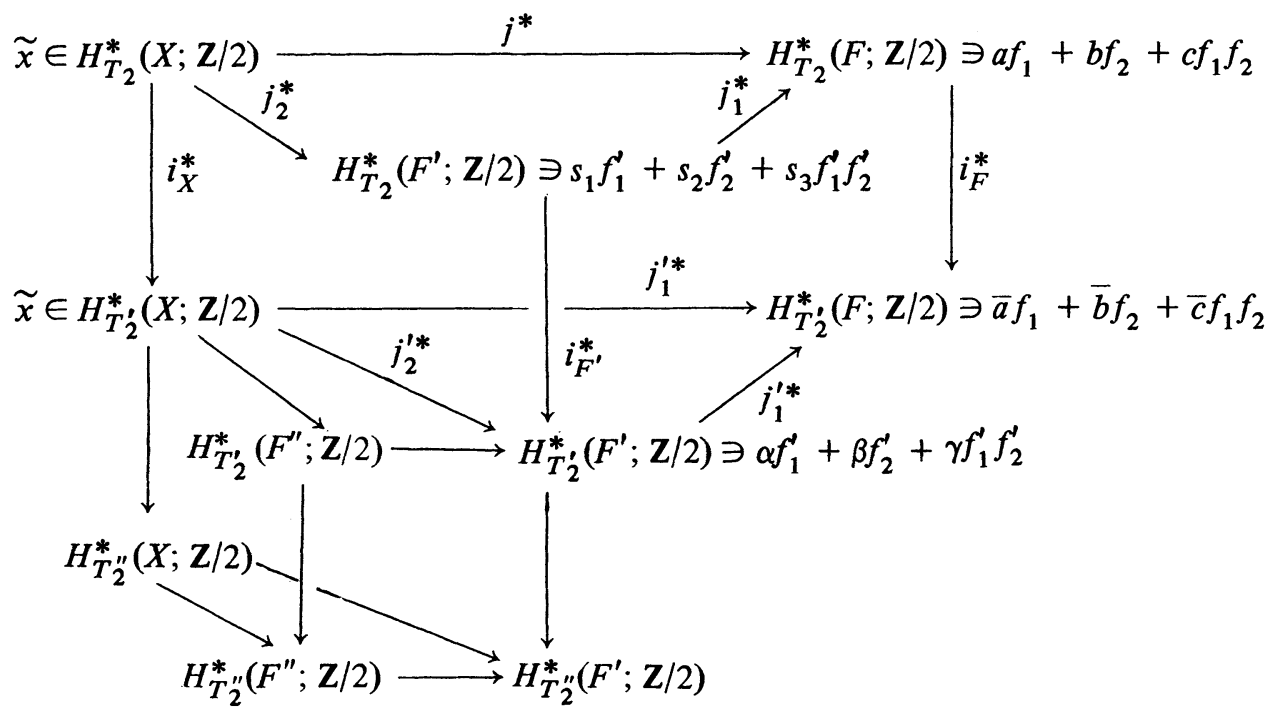

FigURE III

LEMMA 12. In the above setting, $H_{T_{2}}^{*}\left(F^{\prime} ; \mathbf{Z} / 2\right) \approx \Lambda_{R_{T_{2}}}\left(f_{1}^{\prime}, f_{2}^{\prime}\right)$.

Proof. Note that $\operatorname{dim}_{2} H^{*}\left(F^{\prime} ; \mathbf{Z} / 2\right)=4=\operatorname{dim}_{2} H^{*}(F ; \mathbf{Z} / 2)$. Also, $T_{2} / T_{2}^{\prime} \approx \mathbf{Z} / 2$ as well as $T_{2}$ act on $F^{\prime}$ with fixed point set $F$. So by Corollary 2 on p. 46 of [14], the spectral sequence of $F_{T_{2}}^{\prime} \rightarrow B_{T_{2}}$ collapses. (Because we shall prove that $\operatorname{dim} f_{2}^{\prime}-$ $\operatorname{dim} f_{1}^{\prime}=2$ inductively, we are guaranteed that the spectral sequence has simple 
coefficients.) We may then pick lifts of $f_{1}^{\prime}$ and $f_{2}^{\prime}$, the generators of the cohomology algebra $H^{*}\left(F^{\prime} ; \mathrm{Z} / 2\right)$ in the same manner as on pp. 602-603. Now $j_{1}^{*}$ is a monomorphism and the rest follows easily.

We then choose generators of $H_{T_{2}^{\prime}}^{*}\left(F^{\prime} ; \mathbf{Z} / 2\right)$ by taking the image of the generators of $H_{T_{2}}^{*}\left(F^{\prime} ; \mathbf{Z} / 2\right)$. We may assume that $i_{F^{\prime}}^{*}\left(f_{1}^{\prime}\right)=1 \otimes f_{1}^{\prime}$ and $i_{F^{\prime}}^{*}\left(f_{2}^{\prime}\right)=1 \otimes f_{2}^{\prime}$. Note that the vertical maps $i_{X}^{*}, i_{F^{\prime}}^{*}$, and $i_{F}^{*}$ are given in this manner and by $r^{*}$ : $H^{*}\left(B_{T_{2}} ; \mathbf{Z} / 2\right) \rightarrow H^{*}\left(B_{T_{2}^{\prime}} ; \mathbf{Z} / 2\right)$, which sets $t_{m}$ equal to 0 . We denote $r^{*}(a)$ by $\bar{a}$, for example.

Lemma 13. Let $\mathbf{Z} / 2 \approx T_{2} / T_{2}^{\prime}$. Then we have a commutative diagram with injective vertical maps.

$$
\begin{array}{ccc}
H_{T_{2}}^{*}\left(F^{\prime} ; \mathbf{Z} / 2\right) & \stackrel{j_{1}^{*}}{\longrightarrow} & H_{T_{2}}^{*}(F ; \mathbf{Z} / 2) \\
\uparrow & \uparrow \\
H_{\mathbf{Z} / 2}^{*}\left(F^{\prime} ; \mathbf{Z} / 2\right) & \stackrel{\tilde{j}_{1}^{*}}{\longrightarrow} & H_{\mathbf{Z} / 2}^{*}(F ; \mathbf{Z} / 2)
\end{array}
$$

Proof. Straightforward.

Since we had some freedom in choosing the lifts $f_{1}^{\prime}$ and $f_{2}^{\prime}$, we may assume

$$
H_{\mathbf{Z} / 2}^{*}\left(F^{\prime} ; \mathbf{Z} / 2\right) \approx \Lambda_{R_{\mathbf{Z} / 2}}\left(f_{1}^{\prime}, f_{2}^{\prime}\right) \rightarrow \Lambda_{R_{T_{2}}}\left(f_{1}^{\prime}, f_{2}^{\prime}\right) \approx H_{T_{2}}^{*}\left(F^{\prime} ; \mathbf{Z} / 2\right)
$$

is given by $f_{1}^{\prime} \mapsto f_{1}^{\prime}$ and $f_{2}^{\prime} \mapsto f_{2}^{\prime}$.

LEMMA 14. If $m \equiv 0$ (4) and $F \neq \varnothing$, then $F$ is $\mathrm{Sq}^{2}$ linked.

Proof. We begin by exploring when $w_{m}^{k}$ occurs as a term in $\mathrm{Sq}^{2} x$, where $x$ is a symmetric polynomial in $t_{1}, \ldots, t_{m}$. If so, $w_{m}^{k}$ must come from $\operatorname{Sq}^{2}\left(w_{i} A\right)$ where $w_{i} A$ is a term in $x$. Now

$$
\mathrm{Sq}^{2}\left(w_{i} A\right)=w_{2} w_{i} A+\left(\begin{array}{c}
i-1 \\
2
\end{array}\right) w_{i+2} A+\left(\begin{array}{c}
i-1 \\
1
\end{array}\right) w_{i+1} \mathrm{Sq}^{1} A+w_{i} \mathrm{Sq}^{2} A .
$$

$w_{m}^{k}$ clearly cannot come from the first term. If $w_{m}^{k}$ comes from the third term, then $i=m-1$ and $\left(\begin{array}{c}m-2 \\ 1\end{array}\right)=1(\bmod 2)$, which implies that $m$ is odd. If $w_{m}^{k}$ comes from the second term, then $i=m-2$ and $\left(\begin{array}{c}m-3 \\ 2\end{array}\right) \equiv 1(\bmod 2)$. If $m$ is even, the last condition holds iff $m \equiv 2$ (4). Hence if $m \equiv 2$ (4), $w_{m}^{k}$ must come from the last term. By induction we are reduced to the case where $k=1$. From the expression of $\mathrm{Sq}^{2}\left(w_{i} A\right)$, we see this case cannot have any solution.

Suppose now that $F$ is not $\mathrm{Sq}^{2}$ linked. The equation $a \mathrm{Sq}^{2} b+b \mathrm{Sq}^{2} a=w_{m}^{r}$ holds, and some term $x$ in $b$ or $a$ upon application of $\mathrm{Sq}^{2}$ must give rise to a $w_{m}^{k}$ for some $k$. As was just shown this cannot happen.

LEMMA 15. $r$ is even.

Proof. We first remark that $r$ remains unchanged if we restrict our action to $\mathrm{SO}(m-j)$. Since by assumption $\left(r_{0} \geqslant 8\right)$ we may always restrict the action to $\mathrm{SO}(m-j), j=0,1,2,3$, we need only prove the lemma when $m \equiv 0$ (4). In that case, $\mathrm{Sq}^{2} f_{1}=f_{2}$ by Lemma 8 and so the equation is $a^{2}+a \mathrm{Sq}^{2} b+b \mathrm{Sq}^{2} a=w_{m}^{r}$. 
Assume that $a=w_{m}^{k}+$ other terms, then $2 k=r$. So it remains to consider the case when $a$ does not contain $w_{m}^{k}$. In that case, the only term that can give rise to $w_{m}^{r}$ is the term $b \mathrm{Sq}^{2} a$. In other words, some term in $a$ upon squaring must yield $w_{m}^{k^{\prime}}$. By the proof of Lemma 14, we see that this cannot happen for $m \equiv 0$ (4).

REMARK. As a corollary of the fact that $r$ is even, we see that the equation $a \mathrm{Sq}^{1} a+a \mathrm{Sq}^{2} b+b \mathrm{Sq}^{2} a=w_{m}^{r}$ never holds because $2 \operatorname{dim} a+1=m r$, which is even.

We next analyze the case $m \equiv 1$ (4) in greater detail. In view of Lemma 15 we write $r=2 k$.

Case 1: $F$ is $\mathrm{Sq}^{2}$ linked. By calculations similar to those in $\$ 5$ (but more complicated), we obtain that $j^{*}(\tilde{x})=w_{m}^{k} f_{1}+c f_{1} f_{2}$ and the map $H^{*}\left(F^{\prime} ; \mathbf{Z} / 2\right) \rightarrow$ $H^{*}(F ; \mathbf{Z} / 2)$ is zero.

Case 2: $F$ is not $\mathrm{Sq}^{2}$ linked. In this case, again by calculations similar to those in $\S 5, \operatorname{dim} f_{2}-\operatorname{dim} f_{1}=2$ and $k \geqslant 2$. We have $j^{*}(\tilde{x})=w_{m}^{k} f_{1}+w_{m}^{k-2} w_{m-1}^{2} f_{2}+c f_{1} f_{2}$ and the map $\tilde{H}^{*}\left(F^{\prime} ; \mathbf{Z} / 2\right) \rightarrow \tilde{H}^{*}(F ; \mathbf{Z} / 2)$ is 0 unless $k=2$, in which case it is given by $f_{1}^{\prime} \mapsto f_{2}, f_{2}^{\prime} \mapsto 0$.

\section{LEMMA 16. If $m \equiv 2$ (4) then $F$ is $\mathrm{Sq}^{2}$ linked.}

Proof. Note that $F$ is an integral cohomology product of two odd spheres. Suppose first that $F^{\prime}$ is $\mathrm{Sq}^{2}$ linked and $F$ is not $\mathrm{Sq}^{2}$ linked. Then the analysis of Case 2 shows that $a=w_{m}^{k}, b=w_{m}^{k-2} w_{m-1}^{2}$. Recall that $s_{2}=\left(t_{1} \ldots t_{m-1}\right)^{k-2} A$. Now $\bar{s}_{2}=\beta$ $=\hat{w}_{m-1}^{k-2} \hat{w}_{m-2}^{2} \neq 0$. However, since $F^{\prime}$ is $\mathrm{Sq}^{2}$ linked and $m \equiv 2$ (4), it follows from the analysis of Case 1 that $\beta=0$, giving us a contradiction.

Therefore, we shall suppose that $F, F^{\prime}$ are both $\mathrm{Sq}^{2}$ unlinked. Despite this, Lemma 12 shows that $H_{T_{2}}^{*}\left(F^{\prime} ; \mathbf{Z} / 2\right) \approx \Lambda_{R_{T_{2}}}\left(f_{1}^{\prime}, f_{2}^{\prime}\right)$.

Let $j_{1}^{*}\left(f_{1}^{\prime}\right)=u_{1} f_{1}+u_{2} f_{2}+u_{3} f_{1} f_{2}, j_{1}^{*}\left(f_{2}^{\prime}\right)=v_{1} f_{1}+v_{2} f_{2}+v_{3} f_{1} f_{2}$. We have the simplified equation $u_{1} v_{2}+u_{2} v_{1}=t_{m}^{2 k}$. We may assume as in the previous cases that $u_{1}=e_{1} t_{m}^{k_{1}}, u_{2}=e_{2} t_{m}^{k_{2}}, v_{1}=e_{3} t_{m}^{k_{3}}, v_{2}=e_{4} t_{m}^{k_{4}}$. Substituting, we get $e_{1} e_{4} t_{m}^{k_{1}+k_{4}}+$ $e_{2} e_{3} t_{m}^{k_{2}+k_{3}}=t_{m}^{2 k}$. Clearly, exactly one of $e_{1} e_{4}$ or $e_{2} e_{3}$ may be 0 . So there are two cases.

(i) $e_{2} e_{3}=0, e_{1} e_{4}=1$.

We have $a=s_{1} t_{m}^{k_{1}}+e_{3} e_{2} t_{m}^{k_{3}}, b=e_{2} s_{1} t_{m}^{k_{2}}+s_{2} t_{m}^{k_{4}}$. By assumption, $a \neq 0, b \neq 0$; $s_{1} \neq 0, s_{2} \neq 0$ because $\alpha \neq 0, \beta \neq 0\left(\alpha \mathrm{Sq}^{2} \beta+\beta \mathrm{Sq}^{2} \alpha=\hat{w}_{m-1}^{2 k}\right) . k_{1}+k_{4}=2 k$ holds. Recall that $a \mathrm{Sq}^{2} b+b \mathrm{Sq}^{2} a=w_{m}^{2 k}$. If $w_{m}^{2 k}$ comes from $b \mathrm{Sq}^{2} a$, then $\operatorname{dim} a=$ $2 k m-2-m k^{\prime} \geqslant \operatorname{dim} b=m k^{\prime} \geqslant \operatorname{dim} s_{2}=\operatorname{dim} \beta=k(m-1)-2$. Comparing these inequalities we find that $k^{\prime} \leqslant k-1$ and $k^{\prime}>k-1$ simultaneously. Hence $w_{m}^{2 k}$ comes from $a \mathrm{Sq}^{2} b$. In this case $\operatorname{dim} a=k^{\prime} m \geqslant 2 m k-2-\operatorname{dim} a$ and so $k^{\prime} \geqslant k$. Next $\operatorname{dim} b=2 m k-m k^{\prime}-2 \geqslant \operatorname{dim} s_{2}=k(m-1)-2$ implies that $k^{\prime} \leqslant$ $k$. Hence $k_{1}=k=k_{4}$. It follows that $e_{2}=1$; for otherwise $t_{m}^{k}$ would divide $b$ or $w_{m}^{k}$ divides $b$. We have $a=s_{1} t_{m}^{k}$ and so $s_{1}=\left(t_{1} \ldots t_{m-1}\right)^{k}$. We can now use symmetry under the Weyl group to get $a=w_{m}^{k}, b=w_{m}^{k-2} w_{m-1}^{2}$. Let us however compute $a \operatorname{Sq}^{2} b+b \operatorname{Sq}^{2} a=w_{m}^{k}\left(\operatorname{Sq}^{2}\left(w_{m}^{k-2}\right) w_{m-1}^{2}+w_{m}^{k-2} \operatorname{Sq}^{2}\left(w_{m-1}^{2}\right)\right)+w_{m}^{k-2} w_{m-1}^{2} \operatorname{Sq}^{2}\left(w_{m}^{k}\right)$ $=w_{m}^{2 k-2}\left(w_{m}\left(\begin{array}{c}m-2 \\ 1\end{array}\right)\right)^{2}=0$, since $m$ is even. This gives a contradiction.

(ii) $e_{2} e_{3}=1, e_{1} e_{4}=0$. 
We have $a=e_{1} s_{1} t_{m}^{k_{1}}+s_{2} t_{m}^{k_{3}}, b=s_{1} t_{m}^{k_{2}}+e_{4} t_{m}^{k_{4}} s_{2}$. As in (i), we can show that $k=k^{\prime}$ and $k_{3}=k+2, k_{2}=k-2$. Hence $e_{4}=0$ and $e_{1}=1$. Thus $b=w_{m}^{k-2} P$, where $P$ is a symmetric polynomial. $a=w_{m}^{k}$ and $s_{1}=\left(t_{1} \ldots t_{m-1}\right)^{k-2} P$. By a symmetry argument again we see that $a=w_{m}^{k}$ and $b=w_{m}^{k-2} w_{m-1}^{2}$. As in (i), they do not satisfy $a \mathrm{Sq}^{2} b+b \mathrm{Sq}^{2} a=w_{m}^{2 k}$.

Using Lemma 16 and doing computations as above, we get that when $m \equiv 2$ (4), $F$ is always $\mathrm{Sq}^{2}$ linked. If $F^{\prime}$ is $\mathrm{Sq}^{2}$ linked, $j^{*}(\tilde{x})=w_{m}^{k} f_{1}+c f_{1} f_{2}$. If $F^{\prime}$ is $\mathrm{Sq}^{2}$ unlinked, then $j^{*}(\tilde{x})=w_{m}^{k} f_{1}+w_{m}^{k-2} w_{m-1}^{2} f_{2}+c f_{1} f_{2}$. The map $\tilde{H}^{*}\left(F^{\prime} ; \mathbf{Z} / 2\right) \rightarrow$ $\tilde{H}^{*}(F ; \mathbf{Z} / 2)$ is 0 except when $k=2$ and $F^{\prime}$ is $\mathrm{Sq}^{2}$ unlinked, in which case it is given by $f_{1}^{\prime} \mapsto f_{2}, f_{2}^{\prime} \mapsto 0$.

The reader may now check that stage 1 has completely been achieved since the cases $m \equiv 3,4$ (4) are analogous to the cases $m \equiv 1,2$ (4) once we have proved Lemma 16.

We now move on to stage two. We first recall the following theorem of J. C. Su, a proof of which can be found in [23].

THeOREM (J. C. SU). If $\mathbf{Z} / 2$ acts on a $\mathbf{Z} / 2$-cohomology product $X$ of two spheres $S^{m} \times S^{n}$, then the cohomology algebra $H^{*}(F ; \mathbf{Z} / 2)($ where $F=F(\mathbf{Z} / 2, X))$ is given as follows:

(a) if $E_{2} \neq E_{\infty}$ in the Serre spectral sequence of $X_{\mathbf{z} / 2} \rightarrow B_{\mathbf{z} / 2}$, then $F \sim_{\mathbf{z} / 2} S^{p}$.

(b) if $E_{2}=E_{\infty}$, then if $F$ is connected we may have $H^{*}(F ; \mathbf{Z} / 2) \approx$

(i) $H^{*}\left(S^{p} \times S^{q} ; \mathbf{Z} / 2\right), p, q>0$,

(ii) $\mathbf{Z} / 2[z] /\left(z^{4}\right)$,

(iii) $\mathbf{Z} / 2[u, v] /\left(u^{2}=v^{2} \neq 0, u v=0\right) \operatorname{dim} u=\operatorname{dim} v=1,2,4,8$

(c) if $E_{2}=E_{\infty}$ and if $F$ is disconnected then $H^{*}(F ; \mathbf{Z} / 2) \approx$

(i) $H^{*}\left(S^{0} \times S^{0} ; \mathbf{Z} / 2\right)$ or $H^{*}\left(S^{0} \cup S^{p} ; \mathbf{Z} / 2\right), p \geqslant 1$,

(ii) $H^{*}\left(S^{p} \cup S^{q} ; \mathbf{Z} / 2\right), p, q>0$,

(iii) $\mathbf{Z} / 2 \oplus \mathbf{Z} / 2[z] /\left(z^{3}\right)$.

Recall from Lemma 13 that we have a commutative diagram

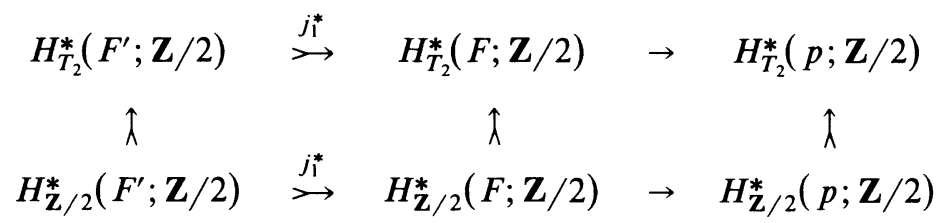

Since $m$ is odd, $m-1$ is even and $F^{\prime} \sim_{\mathrm{z}} S^{p^{\prime}} \times S^{q^{\prime}}, p^{\prime} \leqslant q^{\prime}, p^{\prime}, q^{\prime}$ odd. In fact, by Lemmas 14 and $16, q^{\prime}=p^{\prime}+2$ and $\operatorname{Sq}^{2} f_{1}^{\prime}=f_{2}^{\prime}$, where $H^{*}\left(F^{\prime} ; \mathbf{Z} / 2\right) \approx \Lambda_{\mathbf{Z} / 2}\left(f_{1}^{\prime}, f_{2}^{\prime}\right)$. Let $H^{*}\left(B_{\mathbf{Z} / 2} ; \mathbf{Z} / 2\right) \approx \mathbf{Z} / 2[t]$. We remind the reader of our choice of generators for these cohomology algebras.

RemarKs. 1. Since $\operatorname{dim}_{2} H^{*}\left(F^{\prime} ; \mathbf{Z} / 2\right)=4=\operatorname{dim}_{2} H^{*}\left(F^{\prime} \mathbf{Z} / 2\right), E_{2}=E_{\infty}$ in the spectral sequence for $F_{\mathbf{Z} / 2}^{\prime} \rightarrow B_{\mathbf{Z} / 2}$ and so (a) in Theorem 2 does not occur. 
2. If $q \in F$, the slice representation at $q$ is $r \rho_{m}+$ (trivial). Hence the different components of $F$ have the same dimension. Thus (c)(iii) and (c)(i) $S^{0} \cup S^{p}$ do not occur. For (c)(ii), $p=q$ necessarily.

Claim 1. (c)(i) does not occur.

To see this we apply theorem IV-I in [14], noting that the only homogeneous polynomials in dimension $p^{\prime}$ are 0 and $t^{p^{\prime}}$. Because $\mathrm{Sq}^{2} f_{1}^{\prime}=f_{2}^{\prime}, j_{1}^{*}\left(f_{1}^{\prime}\right)$ completely distinguishes the components of the fixed point set. Hence $F$ can have at most two components.

Claim 2. (c)(ii) does not occur.

We first show that $j_{1}^{*}\left(f_{1}^{\prime}\right)=\left(t^{d} z_{1}, t^{p^{\prime}}+t^{d} z_{2}\right)$, where $H^{*}(F ; \mathbf{Z} / 2) \approx \mathbf{Z} / 2\left[z_{1}\right] /\left(z_{1}^{2}\right)$ $\oplus \mathbf{Z} / 2\left[z_{2}\right] /\left(z_{2}^{2}\right), \operatorname{dim} z_{1}=\operatorname{dim} z_{2}=p, p^{\prime}-p=d$. Since we may assume that the first component of $j_{1}^{*}\left(f_{1}^{\prime}\right)$ has no constant term, it follows that the second component must have constant term $t^{p^{\prime}}$. Next, the ideal $I_{z_{1}}(X, F)$ (see p. 59 of [14]) $=\left(t^{r}\right)$ is nonzero by Theorem IV-10 of [14], and so the first component must have a nonzero $z_{1}$ term. Similarly, the second component must contain a nonzero $z_{2}$ term. $j_{1}^{*}\left(f_{2}^{\prime}\right)=$ $j_{1}^{*}\left(\mathrm{Sq}^{2} f_{1}^{\prime}\right)=\left([d / 2]^{2} t^{d+2} z_{1},\left[p^{\prime} / 2\right]^{2} t^{p^{\prime}+2}+[d / 2]^{2} t^{d+2} z_{2}\right)$. Note that $\operatorname{dim} F^{\prime}=$ $\operatorname{dim} F=2 p^{\prime}+2-p=d+p^{\prime}+2=r \equiv 0$ (4). Hence $p^{\prime}+d \equiv 2$ (4) implies that $p^{\prime} \equiv d \equiv 1(4)$ or $p^{\prime} \equiv d \equiv 3$ (4) ( $p^{\prime}$ is odd and so since $r$ is even, $d$ must be odd). In the first case, $\left[p^{\prime} / 2\right]^{2}=[d / 2]^{2}=0(2)$ and in the second case $\left[p^{\prime} / 2\right]^{2}=[d / 2]^{2}=$ 1(2). Consider

$$
j_{1}^{*}\left(f_{2}^{\prime}\right)=\left\{\begin{array}{l}
(0,0) \quad \text { if } p^{\prime} \equiv d \equiv 1(4), \\
\left(t^{d+2} z_{1}, t^{p^{\prime}+2}+t^{d+2} z_{2}\right)=t^{2}\left(t^{d} z_{1}, t^{p^{\prime}}+t^{d} z_{2}\right) \quad \text { if } p^{\prime} \equiv d \equiv 3(4) .
\end{array}\right.
$$

The first case cannot occur since $j_{1}^{*}$ is a monomorphism. For the second case, $j_{1}^{*}\left(f_{2}^{\prime}\right)=t^{2} j_{1}^{*}\left(f_{1}^{\prime}\right)$, so that $j_{1}^{*}\left(f_{2}^{\prime}-t^{2} f_{1}^{\prime}\right)=0$ or $f_{2}^{\prime}=t^{2} f_{1}^{\prime}$, contradicting the independence of $f_{1}^{\prime}$ and $f_{2}^{\prime}$ over $H^{*}\left(B_{\mathbf{Z} / 2} ; \mathbf{Z} / 2\right)$.

Hence $F$ is connected.

Finally, using similar reasoning, we rule out the cases in (b) of J. C. Su's theorem.

We conclude this section with a discussion of the condition $\mathrm{Sq}^{1} \tilde{y}=0$. At this moment, $\mathrm{Sq}^{1} \tilde{y}$ appears to be the cohomological obstruction to the $\operatorname{SO}(m)$ actions (satisfying the hypotheses of the main theorem) being completely modelled after the regular linear models. We conjecture that $\mathrm{Sq}^{1} \tilde{y}=0$ for any $\mathrm{SO}(m)$ action satisfying the hypotheses of the main theorem. $\mathrm{Sq}^{1} \tilde{y}=0$ is implied, for example, by $\tilde{y}$ being an integral class. If the coefficient system of the $\mathbf{Z}$ Serre spectral sequence of $X_{T_{2}} \rightarrow B_{T_{2}}$ is simple, then $\mathrm{Sq}^{1} \tilde{y}$ would be 0 . In the case of the linear models, 4 divides $r$ because the action results from complexifying $k \rho_{m}(r=2 k)$. So to show that 4 divides $r$ in the general case involves detecting this complexification cohomologically or by some other means.

Note that the $\mathrm{Sq}^{1} \tilde{y}$ obstruction is not unlike that in [21] (or the theorem on p. 58 of [5]) and the discussion of Theorem 2 on pp. 376-377 in [8] may be relevant.

We think that the obstruction is fictitious because in the case of $W_{n, 2}$ much more structure is available from topology. Finally, we remark that we have not been able to remove the $\mathrm{Sq}^{1} \tilde{y}$ obstructions by appealing to the Adem relations. 


\section{REFERENCES}

1. J. F. Adams, Lectures on Lie groups, Benjamin, New York, 1969.

2. M. Atiyah, R. Bott and A. Shapiro, Clifford modules, Topology 3 (1964), 3-38.

3. A. Borel, Sous-groupes commutatifs et torsion des groupes de Lie compacts connexes, Tôhoku Math. J. 13 (1961), 216-240.

4. _ Topics in the homology theory of fibre bundles, Lecture Notes in Math., vol. 36, SpringerVerlag, Berlin and New York, 1967.

5. A. Borel et al., Seminar on transformation groups, Ann. of Math. Studies, No. 46, Princeton Univ. Press, Princeton, N. J., 1960.

6. A. Borel and F. Hirzebruch, Characteristic classes and homogeneous spaces. I, Amer. J. Math. 80 (1958), 458-538.

7. A. Borel and de Siebenthal, Le sous-groupes fermes de rang maximum des groupes de Lie clos, Comment. Math. Helv. 23 (1949), 200-221.

8. G. Bredon, Introduction to compact transformation groups, Academic Press, New York, 1972.

9. __ Homotopical properties of fixed point sets of circle group actions. I, Amer. J. Math. 91 (1969), $874-888$.

10. T. Chang and T. Skjelbred, The topological Schur lemma and related results, Ann. of Math. 100 (1974), 307-321.

11. W. C. Hsiang and W. Y. Hsiang, Differentiable actions of compact connected classical groups. I, Amer. J. Math. 89 (1967), 705-786; II, Ann. of Math. 92 (1970), 189-223; III. Ann. of Math. 99 (1974), $220-256$.

12. Some problems in differentiable transformation groups, Proc. Conf. Transformation Groups, New Orleans, 1967, Springer-Verlag, Berlin and New York, 1968, pp. 223-234.

13. W. C. Hsiang, W. Y. Hsiang and M. Davis, Differentiable actions of compact simple Lie groups on homotopy spheres and euclidean spaces, Proc. Sympos. Pure and Appl. Math., vol. 32, Amer. Math. Soc., Providence, R. I., 1977, pp. 99-109.

14. W. Y. Hsiang, Cohomology theory of topological transformation groups, Ergebnisse der Mathematik und ihrer Grenzgebiete, Band 85, Springer-Verlag, New York, 1975.

15. __ On characteristic classes of compact homogeneous spaces and their application in compact transformation groups. I, Preprint, University of California, Berkeley, 1979.

16. __ On the classification of compact linear groups with non-trivial principal isotropy subgroups, Preprint, University of California, Berkeley, 1979.

17. W. Y. Hsiang and J. C. Su, On the classification of transitive effective actions on Stiefel manifolds, Trans. Amer. Math. Soc. 130 (1968), 322-336.

18. I. M. James, On the homotopy type of Stiefel manifolds, Proc. Amer. Math. Soc. 29 (1971), 151-158.

19. The topology of Stiefel manifolds, Cambridge University Press, London, 1976.

20. I. M. James and J. H. C. Whitehead, The homotopy theory of sphere bundles over spheres, Proc. London Math. Soc. 3 (1954), 196-218.

21. S. D. Liao, A theorem on periodic transformations of homology spheres, Ann. of Math. 56 (1952), 68-83.

22. H. Samelson, Notes on Lie algebras, Van Nostrand-Reinhold, New York, 1969.

23. J. C. Su, Periodic transformation on the product of two spheres, Trans. Amer. Math. Soc. 112 (1964), 369-380.

24. W. Sutherland, A note on the parallelizability of sphere bundles over spheres, J. London Math. Soc. 39 (1964), 55-62.

25. M. Wang, A note on the calculation of Stiefel-Whitney classes and a paper of $W u$ Yi Hsiang, Pacific J. Math.

26. _ Dissertation, Stanford University, 1980.

Department of Mathematics, Stanford University, Stanford, California 94305

Department of Mathematics, University of Pennsylvania, Philadelphia, Pennsylvania 19104 